\title{
Un compte de fabrique de la paroisse Saint- Guénolé de Batz (mars 1478-mars 1479)
}

Alain Gallicé et Gildas Buron

\section{OpenEdition}

\section{Journals}

Édition électronique

URL : http://journals.openedition.org/abpo/1282

DOI : $10.4000 / a b p o .1282$

ISBN : 978-2-7535-1494-2

ISSN : 2108-6443

Éditeur

Presses universitaires de Rennes

Édition imprimée

Date de publication : 20 juillet 2004

Pagination : 29-71

ISBN : 978-2-7535-0031-0

ISSN : 0399-0826

\section{Référence électronique}

Alain Gallicé et Gildas Buron, « Un compte de fabrique de la paroisse Saint-Guénolé de Batz (mars

1478-mars 1479) », Annales de Bretagne et des Pays de l'Ouest [En ligne], 111-2 | 2004, mis en ligne le 20 juillet 2006, consulté le 19 avril 2019. URL : http://journals.openedition.org/abpo/1282 ; DOI :

$10.4000 / a b p o .1282$ 


\title{
Un compte de fabrique de la paroisse Saint-Guénolé de Batz (mars 1478-mars 1479)
}

\author{
Alain GALLICÉ \\ Coordinateur académique patrimoine, Rectorat de Nantes \\ Docteur en histoire médiévale, CRHIA - Université de Nantes \\ Gildas BURON \\ Conservateur, Musée intercommunal des Marais Salants, Batz-sur-Mer
}

L'intérêt que représentent les comptes de fabrique a été souligné par des travaux récents ${ }^{1}$. Plusieurs fois cité, un compte de fabrique de la paroisse de Batz, tenu de mars 1478 à mars 1479, n'a pas encore donné lieu à une étude systématique ${ }^{2}$.

La paroisse de Batz au Moyen Âge comprend les communes actuelles de Batz-sur-Mer, Le Pouliguen et Le Croisic, soit un territoire relativement étendu $\left(17,24 \mathrm{~km}^{2}\right)$ où l'habitat est diversifié, constitué de villages (Kervalet,

1. CONSTANT, abbé Georges, « Une source négligée d'histoire ecclésiastique locale : les registres anciens des marguilliers ", Revue d'Histoire de l'Église de France, 33, 1938, p. 170183; GoUJARD, Philippe, «Les fonds de fabriques paroissiales : une source d'histoire religieuse méconnue », ibid., 68, 1985, p. 99-111; CHIFFolEAU, Jacques, « Pour une économie de l'institution ecclésiale à la fin du Moyen Âge ", Mélanges de l'École française de Rome, Moyen Âge, 1984, p. 247-279; MARTiN, Hervé, "Une paroisse bretonne à la fin du Moyen Âge : Orvault (1460-1520), Kreiz, 1, 1992, p. 135-151; L'argent des villages du XIII au XVII' siècle. Actes du colloque d'Angers (30-31 octobre 1998), dir. FolLAIN, Antoine, Rennes, 2000; LAGADEC, Yann et RESTIF, Bruno, " Pouvoir et religion de Haute-Bretagne au XVI ${ }^{\mathrm{e}}$ siècle : le compte de fabrique de Louvigné-de-Bais (1503-1504) ", Annales de Bretagne et des Pays de l'Ouest, 190, 2002, p. 31-60. Voir encore dans une perspective incluant les villes, PERRAUD-CHARMANTIER, André, Essai sur le général de la paroisse en Bretagne particulièrement dans le diocèse de Nantes, et dans le dernier état de l'institution, Thèse de droit, Rennes, 1926; Minois, Georges, "L'administration paroissiale dans le Trégor au xve siècle ", Société d'émulation des Côtes$d u$-Nord, 107, 1978, p. 59-79; id., "Culte des saints et vie religieuse dans le diocèse de Tréguier au Xve siècle ", Annales et Bretagne et des Pays de l'Ouest, 78, 1980, p. 17-42.

2. MonNIER, Pierre-Aristide, Le pays guérandais. Exploration du pays guérandais primitif. Études sur l'île guérandaise de Batz (comprenant Le Pouliguen, Batz et Le Croisic), 2 vol. Angers 1897, t. 1, p. 209-210, avec une date fautive de 1428 et d'autres erreurs de lecture; BoURDEAUT, A., La chapelle de Notre-Dame-du-Murié au Bourg de Batz, Fontenay-le-Comte, 1935; et surtout BuRON, Gildas, Histoire et patrimoine culturel de Batz-sur-Mer, Batz, 1999, p. 9-13. 
Trégaté, Roffiat, Olonne, Penchâteau...), d'un bourg - Batz, qui est le cheflieu de paroisse -, et d'une ville, Le Croisic. Difficile de se prononcer sur le chiffre de population mais une estimation, pour l'année 1500, donne 4000 habitants pour la seule ville du Croisic et ses villages environnants, chiffre qui pourrait être retenu à titre d'hypothèse pour l'ensemble de la paroisse en 1478/1479. La présence d'une ville et de densités relativement importantes reposent sur la mise en valeur des marais salants et les échanges qu'elle suscite, mais encore sur l'activité d'une flotte engagée dans le commerce roulier. Le développement de ce commerce roulier est avant tout le fait du Croisic et de ses marchands mariniers dont l'affirmation est remarquable au cours du Xve siècle $^{3}$.

Le compte se présente sur la forme d'un cahier papier quelque peu endommagé : le début du document manque, de même que quelques folios, et l'humidité a rendu le contenu de certains articles illisible. S'y ajoutent des quittances intercalées correspondant à certaines pièces comptables présentées par les procureurs ${ }^{4}$.

Le compte commence le 20 mars 1478, c'est-à-dire le Vendredi Saint, mais les comptables ne se chargeant pas de la recette de ce jour, effectuée par les précédents procureurs, leurs recettes débutent à la date du 22 mars, le jour de Pâques pour s'achever le 14 mars 1479. Le compte est conclu le 12 juin 1479, pour les charges, et le 20 juin 1479, pour les mises, soit un délai de près de trois mois entre la fin de la charge et la reddition du compte. Ce délai relativement important s'explique par l'achèvement d'une campagne de travaux engagée à l'église Saint-Guénolé dont attestent des quittances qui se suivent jusqu'en avril 1479. Saint-Guénolé est l'église paroissiale. Elle est le cadre de l'action de la fabrique, organisme financier, dirigé par des laïcs, chargé de veiller à la conservation de l'édifice et du mobilier cultuel d'une église et d'assurer l'administration temporelle de la paroisse. Cependant, la fabrique de Batz a également en charge l'église Notre-Damedu-Mûrier ${ }^{5}$.

3. TANGUY, Bernard et LAGRÉE, Michel (dir.), Atlas d'Histoire de Bretagne, p. 78-79; BuRON, Gildas, Bretagne des marais salants. 2000 ans d'histoire, 2 vol., Morlaix, 1999-2000; GALLICÉ, Alain, Guérande au Moyen Âge. Guérande, Le Croisic, le pays guérandais du milieu du XIVe siècle au milieu du XVI siècle, Rennes, 2003, p. 353-358; id., "Les marchands mariniers du Croisic (vers 1450-vers 1540)", Mémoires de la Société historique et archéologique de Bretagne, 2003, p. 171-204

4. Arch. dép. de Loire-Atlantique, G 632. Pour cette paroisse, il faut ensuite attendre près d'un siècle pour trouver un document de ce type : un autre compte couvre la période du 5 mars 1575 au 24 mars 1576; $1 \mathrm{~J} 134$.

5. Sur l'origine de cette chapelle qui remplace un ancien monument dédié à la Vierge, DenIFLE, abbé Henri, La désolation des églises, monastères et hôpitaux en France pendant la guerre de Cent ans, 2 vol. Paris, 1897, t. p. 122; BOURDEAUT, A., La chapelle ..., op. cit., p. 28. Une épidémie de peste en 1438 est à l'origine de sa reconstruction pour laquelle une bulle, de juillet 1442 donnée par le pape Eugène IV, accorde des indulgences afin d'aider à sa réalisation en réponse à une supplique de Jean V. Le 8 décembre 1496, un bref cardinalice accorde de nouvelles indulgences afin de procurer à la chapelle des livres, calices et ornements, LEGROS, H.-M. et KERCHNER, É., " Lettres d'indulgences de la cour de Rome 
La structure de ce compte est classique : présentation, charges, mises, déduction, régularisation de la situation comptable, passation de pouvoir et serment des nouveaux nommés. Quelques articles traitent de demandes de dégrèvements relatives à certaines charges. Nous présenterons les hommes, les charges, les mises, avant d'envisager les éléments qui concernent la vie religieuse d'une part, la société et l'économie d'autre part.

\section{Les hommes}

Le folio de présentation du compte ayant disparu, des mentions éparses permettent de découvrir les noms des procureurs. À Jacquet Vinces et Guillo Cazdre succèdent Jean Cabelguen et Bonabès Jouhanno, les auteurs du compte, auxquels succéderont Pezdron Le Callouc'h (ou Le Calloc'h) et Jacob Le Laisour.

Jacquet Vinces est sans doute ce Jacques Vinces qui en mars 1493 rend aveu pour une maison rue de l'Hôpital au Croisic. De Guillo Cazdre, on connaît une maison, citée dans le compte, qui a été la sienne au bourg de Batz. Jean Cabelguen est à rapprocher de la mention d'une maison Cabelguen signalée en juin 1460 à Olonne, au Croisic; Bonabès Jouhanno quant à lui est à rapprocher de Thomas Jouhanno qui rend le 8 avril 1467 aveu pour une maison et son courtil à Trégaté, pour des terres, plus de trois journaux, des prés, plus de deux journaux, et deux planches de vignes, et encore de dom Éon Jouhanno qui réside à Batz en mai 1475 et en février 1478. Pezdron Le Callouc'h est cité en 1491 à Penchâteau à propos d'une rente due par Jean Le Callouc'h, ce dernier possédant de son vivant, avant 1470, des œillets de marais; ils sont encore à rapprocher de Guillaume Callouc'h qui rend aveu le 23 octobre 1475 pour une maison rue de l'Hôpital au Croisic. Jacob Le Laisour est le $1^{\text {er }}$ juillet 1480 plege (caution) de Geffroy le Goyc, plus tard par testament il fonde une chapellenie Saint-Jacques d'une messe par semaine qu'il dote de huit œillets de salines, alors qu'une maison Nicolas Le Laizour est signalée au bourch en 1452, et que ce dernier est cité dans le cours du compte pour le prêt d'une haussière (cordage); Olivier Le Laysour est également cité dans le compte et Étienne Le Laisour est mentionné en mai $1480^{6}$.

Incontestablement, ces personnes ne sont pas de tout premier plan, elles ne résident pas toutes au chef-lieu de paroisse, mais paraissent, elles et leur famille, avoir des biens et donc le répondant financier nécessaire.

au Xve siècle ", Revue des études historiques, 1933, p. 543-556. Le vocable de cette chapelle paraît lié à un culte rendu aux Vierges d'arbres et son vocable est donc bien Notre-Damedu-Mûrier, Provost, Georges, La fête et le sacré. Pardons et pèlerinages en Bretagne aux XVII et XVIII siècles, Paris, 1998.

6. Arch. dép. de Loire-Atlantique, B 1447, 19 mars 1493, 24 juin 1460, 8 avril 1467, 27 mai 1475, 2 février 1478 ; B 1490, f $113 \mathrm{v}^{\circ}$; B 1443, 16 octobre 1470, 23 octobre 1475; B 123; Arch. presbytère de Batz-sur-Mer; Arch. dép. de Loire-Atlantique, B 1489 (A), f ${ }^{\circ} 92$; B 1443, 3 mai 1480. 
La présence de deux procureurs pour un mandat d'un an qui paraît non renouvelable est classique. Cependant, si la présence des deux procureurs agissant en commun est mentionnée lors de la passation d'un marché, les quittances conservées n'impliquent que Jean Cabelguen. C'est lui encore, et lui seul, qui se déplace à Nantes pour acheter des matériaux, vraisemblablement pour la campagne de travaux concernant l'église paroissiale Saint-Guénolé. La nomination du procureur prendrait alors en compte une compétence particulière en fonction d'une tâche à accomplir. Le fait que Jean Cabelguen s'adjoigne un couvreur pour choisir un lot d'ardoises ne plaide pas pour une telle hypothèse. L'explication pourrait être trouvée dans le fait que certains procureurs paraissent entrer en fonction relativement jeunes - Jacquet Vinces et Pezdron Le Calloc'h semblent encore en vie au début des années 1490; pour eux une nomination serait une promotion, gage d'expérience, de notabilité et d'intégration à «l'élite " paroissiale. Ainsi, certaines années au moins, un procureur pourrait-il être plus expérimenté que l'autre, et exercer plus de responsabilités.

Un vicaire est cité, il est désigné par le prieur du prieuré conventuel de Batz relevant de Landévennec ${ }^{7}$. Ce vicaire, qui est le commissaire désigné lors de la reddition du compte, est Nicolas Le Goyc, cité également à ce poste par une autre source ${ }^{8}$. Un segretain (sacriste), dom Guillaume Le Bourdiec, est mentionné, il doit faire la cire des deux églises, Saint-Guénolé et Notre-Dame-du-Mûrier, faire lavanderies des ornemenz, et fournir les hosties (en fait simplement les préparer). Dom Raoul Bellego est commis pour tenir la liste des inhumations qu'il présente le 18 avril 1479. Il assure aussi le pointage des maçons, des charpentiers et des couvreurs intervenant lors des travaux entrepris à l'église Saint-Guénolé. Dom Éon Jouhanno est le clerc du vicaire, associé à Jean Onan, il passe marché pour la sonnerie des cloches.

D'autres personnes sont citées : Benjamin du Pont est chargé de la sonnerie des orgues; Olivier Le Pennec porte la bannière et lève le devoir de la robe des morts. Le compte mentionne encore le nom d'un certain nombre de témoins sur lesquels nous reviendrons.

\section{Les charges ${ }^{9}$}

Les charges commencent classiquement par l'enregistrement des trésors. Les nouveaux procureurs sont mis en possession des biens et thre-

7. Il dispose de six religieux selon un procès-verbal de la seconde moitié du xvI ${ }^{\mathrm{e}}$ siècle, OGÉE, Dictionnaire historique et géographique de la province de Bretagne, nouv. éd. par MARTEVILLE, A. et VARIN, P., 2 vol., Rennes, 1843, t. I, p. 96 . Aveu est rendu au duc le 7 août 1475, par Jean de Kerguz, prieur de Batz, Arch. dép. de Loire-Atlantique, B 777; sur la carrière de ce dernier qui sera le principal chapelain de François II, POCQUET Du HAUT-Jussé, Barthélémy-Anatole, Les papes et les ducs de Bretagne. Essai sur les rapports du Saint-Siège avec un État, 2 vol., Paris, 1928, p. 590, 609.

8. Arch. dép. de Loire-Atlantique, B 1489 (A), $\mathrm{f}^{\circ} 64$.

9. Arch. dép. de Loire-Atlantique, G $632, \mathrm{f}^{\circ} 1-13 \mathrm{v}^{\circ}, 25 \mathrm{v}^{\circ}$. 
sors comme ornements, calices, images d'or et d'argent, livres et autres biens, mais il n'est recensé qu'une partie de ce patrimoine : les pièces en argent ou garnies d'argent. On dénombre : une chapelle, quatre croix dont deux petites, un bénitier et un aspersoir (sans doute un seau à eau bénite), deux tasses, deux chandeliers, au moins quatorze calices et leurs plataines (patènes) le plus souvent dorés et émaillés ${ }^{10}$, deux encensoirs avec leurs chaînes et boucles, une pecine, un tableau, une teste, une petite tourelle en forme de chandelier, et encore, en une poche, plusieurs pièces d'argent provenant de la garniture des images. Il n'est pas fait état des poids des pièces sauf pour les tasses qui pèsent chacune un marc d'argent ou environ. Dans cette liste figure encore deux petits orseulz (récipients ${ }^{11}$ ), mais leur mention paraît rapportée et doit correspondre au deux orseulz achetés durant l'année du compte.

Certaines pièces sont décrites avec un soin particulier : l'une des petites croix présente des pierreries au bout et au milieu; les deux petits orseulz sont vere $z^{12}$. Mais l'effort de description porte surtout sur les défauts présentés par les pièces : il s'agit pour les procureurs et le sacriste face aux contrôleurs de n'être pas pris en faute pour mauvais entretien. Ainsi, la chapelle d'argent a-t-elle des suaires en reliquaires sur quatre tourelles et un clocher, mais il manque deux croix aux bouts des tourelles, une croix et une guyrouette sur le clocher, et deux autres girouettes sur deux autres tourelles. Sur la croix, sans doute la grande croix de procession, figurent d'un côté les quatre Évangélistes, le crucifix tout entier avec le diadème et le tiltre, et de l'autre côté les quatre Docteurs de l'Église, dorés, et un Agnus Dei, doré également, avec un pommeau à six pans et sur chacun une image d'argent dorée. Mais sur ce pommeau, en deux des pignons, manquent trois petites croix, de même qu'au pied de la croix et aussi au-dessus du pommeau, aux deux jointures, de la garniture décorée de fleurs de lys, ainsi qu'aux deux bras de chaque côté et en deux endroits au haut d'une des fleurs le lys. Aux deux chandeliers manquent deux petits rolletz d'argent au pied de ceux-ci; à la pecine d'argent doré, il y a un défaut au-dessus de l'émail et partie des feuillages. Enfin, une des patènes n'est pas dorée.

Si la déclaration ne fait pas état du linge, ni des livres, le patrimoine n'est vraisemblablement pas moins important en quantité et qualité, traduisant l'importance de la paroisse et sa richesse relative ${ }^{13}$.

10. Une patène est un vase sacré en forme de petite assiette qui sert à couvrir le calice et à recevoir l'hostie D'après le décret de Gratien, les calices et leurs patènes doivent être d'or ou d'argent avec du vermeil à l'intérieur de la coupe. Cependant, dans les trois premiers quarts du Xve siècle l'étain et le cuivre remplacent l'or et l'argent, LE Duc, A., "Orfèvres et crise à la fin du Moyen Âge ", dans Artistes, artisans et production artistiques en Bretagne au Moyen Âge, BARRAL I AlLET, Xavier (dir.), Rennes, 1983, p. 77. Présentation des objets religieux, Thesaurus des objets religieux. Meubles, objets, vêtements et instruments de musique, Paris, 1999.

11. Ils sont sans doute destinés à conserver les saintes huiles.

12. Terme désignant un effet décoratif obtenu en mêlant de l'or et de l'argent.

13. Comparaisons possibles dans l'évêché de Nantes avec des comptabilités de village : Orvault, Arch. dép. de Loire-Atlantique, 114 J 8, 9-12, à partir de 1460, MARTiN, Hervé, « Une 
La charge en deniers déclarée est de $730112 \mathrm{~s} 7 \mathrm{~d}$. Cette somme inclut le reliquat du compte précédent, $470 \mathrm{l} 11 \mathrm{~s} 5 \mathrm{~d}$, ainsi l'ensemble des recettes de l'année s'élèvent-elles à $26011 \mathrm{~s} 2 \mathrm{~d}$. L'ampleur du reliquat est à souligner, il représente $175 \%$ de la recette de l'année. Les travaux qui vont être réalisés au cours de l'année ont-ils été anticipés par une imposition extraordinaire qui a gonflé momentanément ce poste? Sans doute faut-il plutôt $\mathrm{y}$ voir le signe de la richesse de la paroisse et de la bonne santé financière de la fabrique qui permettent des "investissements".

Les recettes proviennent de plusieurs sources et d'abord des offrandes. La valeur de celles-ci est exprimée en monnaie de compte sans qu'il soit fait référence à la monnaie réelle en circulation, ni à la nature réelle des dons effectués, sauf cas particulier. Certaines offrandes sont faites au tablier ou à la table des églises Saint-Guénolé ou Notre-Dame-du-Mûrier. Pour le tablier de Saint-Guénolé, le document distingue pour le jour de Pâques et les féries de Pâques - le lundi, le mardi et le mercredi suivants - deux types d'offrandes : les offrandes proprement dites et celles reçues sur la table de ceux qui ont fait leurs Pâques ( $18 \mathrm{~s} 9 \mathrm{~d}$ pour Pâques et ses féries, $3 \mathrm{~s}$ pour le 5 avril). À la fête de la Chandeleur le produit des chandelles est comptabilisé avec les offrandes. Ces dernières s'élèvent à $53 \mathrm{l}$ 15 s 7 d (65,3\%, les deux tiers, provenant de Saint-Guénolé). Les revenus provenant des troncs et boîtes de Notre-Dame-du-Mûrier ouverts le 29 mars (y compris, ce jour-là, le produit de deux boîtes de Saint-Guénolé), le 6 juillet, et le 6 novembre, rapportent plus de $77113 \mathrm{~s} 7 \mathrm{~d}$. Des dons en argent ou en nature sont encore enregistrés : $10 \mathrm{~s}$ plus une truellée de froment évaluée à $5 \mathrm{~s}$, donnés par Jean Jouan; $10 \mathrm{~s}$ pour quatre aunes de bougrain de l'église utilisées lors du service mortuaire et de l'inhumation d'Éon Moro, et $1 \mathrm{l}$ pour avoir mis de la cire et l'avoir récupérée pour l'église. Le total des offrandes, tel qu'il apparaît, s'établit à 133 l 14 s 2 d, soit $51,4 \%$ des recettes déclarées. Ce chiffre doit être revu à la hausse. En effet, les relevés concernant les troncs et boîtes de Notre-Dame-du-Mûrier ne dépassent pas le mois de novembre, nous ne disposons que d'une indication concernant Saint-Guénolé, et d'autre part le total de l'ensemble des recettes à partir des déclarations des procureurs est inférieur de $12 \%$ à ce que l'on obtient par calcul à partir de la déclaration de leur charge défalquée du reliquat du compte précédent. Aussi l'ensemble de ce chapitre pourrait-il s'élever à $166110 \mathrm{~s} 9 \mathrm{~d}$, soit $64 \%$, et près des deux tiers des recettes totales.

paroisse... ", op. cit.; Basse-Goulaine, Arch. dép. de Loire-Atlantique, E, dépôt 3 supplément, 3549-3552, à partir de 1473. Et de villes : Saint-Martin-de-Chantenay, Arch. mun. Nantes, GG 17-19, à partir de 1481, LA Nicollière-TeIJERO, Stéphane, " Comptes de la fabrique Saint-Martin de Chantenay (1481-1506) ", Revue de Bretagne et de Vendée, 1875, p. 174185 ; registres de Saint-Nicolas et Sainte-Croix de Nantes. Un inventaire des sources à été publié par HILDESHEIMER, F., Répertoires provisoires des délibérations et comptabilités communales. Moyen Âge et Ancien Régime, 2, Région Pays de la Loire et Poitou-Charente, Paris, 1981 qu'il convient d'actualiser. 
Une autre ressource provient de la tarification d'un service religieux, les inhumations effectuées dans les églises Saint-Guénolé ou Notre-Damedu-Mûrier rapportent 61 l, 23,4 \% des recettes.

Les produits d'impositions sur les paroissiens ont pour origine d'une part la levée de la robe des morts qui consiste en la levée d'une robe, la meilleure - ce qui implique que le décédé en ait plusieurs -, et rapporte 61 19 s 6 d. D'autre part, le denier de Pâques a pour taux 5 d par écuelle, reçu par le sacriste, il produit $615 \mathrm{~s} 5 \mathrm{~d}$. Cette dernière taxe a évolué. À une ancienne imposition de $3 \mathrm{~d}$ par écuelle dont une maille, appelée maille de confession, destinée au prieur, alors que $2 \mathrm{~d}$ maille sont affectés aux gages du sacriste, soit 3 l, ont été ajoutés 2 d par écuelle pour la sonnerie des orgues. Mais la charge du sacriste étant devenue plus importante que par le passé, en particulier en raison de la garde de la chapelle Notre-Dame-duMûrier - ce qui confirme son caractère récent - il a été ordonné de verser $60 \mathrm{~s}$ sur les $2 \mathrm{~d}$ nouvellement imposés. Ces prélèvements, qui assurent $13 \mathrm{l}$ $4 \mathrm{~s} 11 \mathrm{~d}, 5 \%$ des revenus de la fabrique, attestent la possibilité et la capacité de la communauté paroissiale à s'imposer. Elle le fait sur des modes différents. Le denier de Pâques est d'un montant uniforme, alors que la robe des morts tient compte des situations de chacun : ceux qui n'avaient qu'une robe et étaient pauvres ne contribuant pas, et pour ceux qui contribuent, les valeurs des robes sont différentes selon les estimations qui en sont faites.

Les rentes, 34 occurrences, rapportent plus de 10 l 4 s 9 d, l'exploitation de 3 œillets 3114 s 1 d, et la location de deux étals au Croisic 19 s 2 d. Soit un total de $14118 \mathrm{~s}, 5,7 \%$ des recettes totales. Cependant, une des rentes, d'une valeur de $20 \mathrm{~s}$, est destinée à payer les frais à venir de l'inhumation de la veuve Éonnet Herault et celle-ci étant toujours en vie, la rente est impayée ${ }^{14}$.

Enfin, des matériaux de récupération provenant du chantier de l'église sont cédés : des ardoises, à Rio Talic, pour $4 \mathrm{l}$; des vieilles gouttières, à dom Jean Le Maller, pour $7 \mathrm{~s} 6 \mathrm{~d}$. Ces ressources occasionnelles sont d'ampleur limitée, 1,6\% des ressources totales.

Ces sources de recettes sont traditionnelles. À Batz, elles reposent avant tout sur la contribution volontaire des paroissiens. Rapportées au nombre d'écuelles concernées par le denier de Pâques, les offrandes correspondraient à des versements moyens de $12 \mathrm{~s}$. Elles permettent de dégager des revenus importants et d'engager des travaux sans recourir à une taxation exceptionnelle.

\section{Les $_{\text {mises }}{ }^{15}$}

L'état du document ne permet d'établir qu'en partie le total des mises. Il dépasse 220 l en ne prenant en compte que les chiffres lisibles. Le dernier folio,

14 Cette veuve rend aveu le 18 août 1475 sous son nom de jeune fille, Guillemette Elyot, pour une maison et courtil au Croisic, Arch. dép. de Loire-Atlantique, B 1447.

15. Arch. dép. de Loire-Atlantique, G $632, \mathrm{f}^{\circ} 14-26$ et quittances intercalaires. 
très dégradé, paraît indiquer que le reliquat du compte s'établit à $460 \mathrm{l}$, s'il en est ainsi, le total des mises de 1478/1479, se situerait à $270 \mathrm{l}$. La différence est importante : elle peut s'expliquer par le caractère incomplet du document, ou encore un apoinctement passé entre la fabrique et le prieur que suggèrent des bribes de phrases. Quel que soit le chiffre retenu, il est important, mais n'est pas exagéré si on le compare aux recettes de l'année. Apparemment, les projets sont en rapport avec les possibilités de financement.

Le paiement de rentes est un poste limité : $12 \mathrm{~d}$ pour les 2 œillets de la saline Miroudrouc relevant de la seigneurie de Campsillon.

Les frais administratifs comportent la lettre de procuration de la fabrique. Les procureurs déclarent $25 \mathrm{~s}$ pour celle-ci, mais les contrôleurs ne retiennent que $17 \mathrm{~s} 6 \mathrm{~d}$ se référant à un edit et ordonnence de l'évêque de Nantes qui n'autorise pas une allocation supérieure pour seau et escripture.

D'autres dépenses sont liées à la rétribution de services rendus. Il est versé $10 \mathrm{~s}$ à Éon Jouhanno, clerc du vicaire, qui est allé à Nantes chercher la procuration; 15 s, à celui qui a rédigé le compte; 1 l, à dom Raoul Bellego, pour avoir fait la liste des enterrements, celle du comptage des charretées de pierres acheminées, et celle des maçons, couvreurs et charpentiers ayant participé aux travaux accomplis à l'église de Saint-Guénolé; 1 l, à deux serviteurs qui ont gardé les tabliers des églises et en ont reçu les deniers; 15 s, à Olivier Le Pennec pour avoir porté la bannière et perçu le devoir de la robe des morts. D'autres dépenses concernent le défraiement des témoins requis lors de la visite de Monseigneur de Nantes, et les déplacements hors de la paroisse de l'un des procureurs - ce sont là les seules indemnités auxquelles ils peuvent prétendre, leur charge étant bénévole.

Des mises concourent à l'exercice du culte et à sa solennité. Pour la prédication du Carême, il est fait appel à frère François Dignan, dominicain du couvent Saint-Yves de Guérande, lequel reçoit 3 l pour ses prêches. Pour la sonnerie des cloches, il est dépensé $2110 \mathrm{~s}$, pour celle des orgues, $10 \mathrm{l}$, pour de l'encens, 1 l. D'autres frais concernent la pose et dépose de la tapisserie de l'église, et les processions qui marquent les fêtes du Saint Sacrement et de la Trinité engagent encore quelques sous. Les achats de cire, de pain pour le pain bénit, ou encore de vin pour communier à Pâques, articles classiques des comptes de fabrique, n'apparaissent pas sans doute en raison de l'état du document ou de rentes spécifiques non enregistrées.

Des ornements sont achetés : 12 ceintures à saindre les chapelains à la messe, $5 \mathrm{~s} ; 2$ petits orceulz, $10 \mathrm{l}$. La même somme est consacrée à l'achat d'étoffe pour faire une chasuble, une dalmatique, une écharpe, neuf étoles et des fanons ${ }^{16}$. Dix livres sont encore dépensées pour acheter des orfrais

16. Chasuble : vêtement porté par-dessus tous les autres par le prêtre lorsqu'il dit la messe. Dalmatique : vêtement porté par-dessus tous les autres par le diacre et le sousdiacre lors de la messe. Étoles : longue bande d'étoffe portée autour du cou par le célébrant et les desservants lors de la messe et pour administrer les sacrements. Fanons : manipule, bande d'étoffe portée sur le bras gauche par le célébrant et les desservants lors de la messe. 
(passementerie d'or et d'argent) que Pierre Gledel fait venir de Rennes et qui sont destinés à orner ces pièces textiles. Pierre Gleder fournit, toujours à partir de Rennes, encore seize aunes de bougrain de Paris pour leurs doublures, facturées $3 \mathrm{l}$, et du fil noir pour les coudre, $5 \mathrm{~s} 3 \mathrm{~d}$; du fil pour faire les croix des étoles et fanons, $2 \mathrm{~s}$; enfin d'autres dépenses de fil et pour la façon des pièces s'élèvent à $311 \mathrm{~s}$.

Plus importantes sont les sommes consacrées aux travaux accomplis à l'église Saint-Guénolé ${ }^{17}$. Dans le compte, chaque intervention d'un corps de métier fait l'objet d'une déclaration particulière et comporte parfois plusieurs paragraphes. La présentation de chacune de ces interventions suit l'ordre chronologique de leur accomplissement dans la campagne de travaux entreprise. Sont versés $1116 \mathrm{~s} 2 \mathrm{~d}$ pour une goutiere de pierre de taille, longue de 59 pieds et demi, placée entre les nefs de l'église. Cette somme est d'ailleurs un reste à payer d'un marché se montant à $28110 \mathrm{~s} 7 \mathrm{~d}$ passé par les procureurs précédents, le versement antérieur pouvant correspondre à une avance ou au paiement partiel du travail entrepris. Les travaux de maçonnerie sous la gouttière, y compris les fournitures et le transport, s'élèvent à plus de $2619 \mathrm{~s}$. Ces travaux donnent lieu à des quittances qui s'échelonnent du 4 mai au 4 septembre 1478. Le chantier engagé concerne aussi la charpente. Pour faire et asseoir chevrons et coyaux de part et d'autre de la gouttière de pierre, faire les bois pour les noues, il en coûte $8 \mathrm{l}$, une quittance du paiement de cette somme étant rendue le 9 août 1478. La pose de deux lucarnes au-dessus de la gouttière est réalisée pour $7110 \mathrm{~s}$ et le marché, passé le 21 juillet 1478, précise que chacune aura de lese (côté) l'espacement de trois chevrons de l'église et une hauteur permettant de les vitrer et les lambrisser. L'intervention est achevée avant le 13 août 1478 . Les travaux se poursuivent au niveau de la toiture pour laquelle sont engagés $11113 \mathrm{~s} 2 \mathrm{~d}$, montant qui constitue le poste de dépenses le plus important. Il en coûte encore $1110 \mathrm{~d}$ pour l'échafaudage nécessaire aux travaux de maçonnerie et de charpenterie; pour le guindage (hisser à l'aide d'un treuil) des pierres et le bois, on dépense $13 \mathrm{~s} 9 \mathrm{~d}$ pour une corde qui est ensuite réemployée es cloches, alors que pour les plus grosses pierres destinées à la gouttière, on emprunte une haussière et pour dédommagement de son deché il est versé $10 \mathrm{~s}$. La visite de l'évêque de Nantes conduit à des travaux de nettoyage de l'église et du cimetière et c'est peut-être à cette occasion qu'est reprise une brèche apparue au cimetière, reprise facturée $5 \mathrm{~s}$. Les travaux se poursuivent. Il faut encore asseoir la verrine (vitrail) d'une des lucarnes située sur la gouttière, soit une facture de $4 \mathrm{l} 10 \mathrm{~s}$, et affecter plus d'1 l pour les verges et garnitures mises aux vitres de deux lucarnes, quittance du 10 novembre 1478. Un autre vitrail est mis au lovier (lucarne) qui est l'endroit du letrin, ce qui coûte $10 \mathrm{~s}$ et $3 \mathrm{~s}$

17. Sur cette question, MATZ, Jean-Michel, "La reconstruction des églises paroissiales après la guerre de Cent Ans : le témoignage des comptes du diocèse d'Angers ", dans Prigent, Daniel et Tonnerre, Noël-Yves (éd.), La construction en Anjou au Moyen Âge, Actes de la table ronde d'Angers, 1996, Angers, 1998, p. 125-140. 
$9 \mathrm{~d}$ pour le couvreur. Il faut encore lembruncher les lucarnes, entre les noues; il est dépensé $311 \mathrm{~s} 1 \mathrm{~d}$ pour les matériaux et le transport, et intervenir à l'intérieur pour lembruncher la nef et la chapelle Notre-Dame, 6 l 10 s, le marché est signé le 12 février 1479. Les procureurs fournissent les clous, pour $6 \mathrm{~s} 8 \mathrm{~d}$, quittance du 26 mars 1479, et les lambris qu'ils achètent à Nantes, à Saint-Julien, selon une quittance du 24 avril 1479.

À ces travaux, il faut ajouter la réparation de la fenêtre de la grande vitre au-dessous la voûte, par-dessus la grande vitre, et celle d'un panneau de la vitre de la chapelle Notre-Dame où apparaissait un pertuy, ce qui exige la pose d'un panneau de verre neuf $(11 \mathrm{~s} 8 \mathrm{~d}$, quittance du 8 novembre 1478). Enfin, une corde est mise à la petite cloche, $3 \mathrm{~s} 10 \mathrm{~d}$. Ces dernières interventions sont de nature différente des précédentes, elles relèvent de l'entretien courant.

L'absence d'intervention à Notre-Dame-du-Mûrier est notable. L'église construite récemment n'exige-t-elle pas de travaux? Ou ceux-ci sont-ils pris en charge de façon indépendante?

L'état du document ne permet pas de lire les chiffres de la déduction. Le compte se prolonge par des demandes des procureurs, à hauteur de $3 \mathrm{l}$, au sujet d'articles déclarés en charge mais impayés. Ces articles équivalent aux demandes exprimées, dans les comptes d'alors, par les comptables afin d'obtenir une décharge, ce qui peut donner lieu à la mise en deport de certaines sommes, et éventuellement déboucher sur une procédure d'apurement à une date fixée. Ici, la procédure est immédiate. Elle traduit la proximité, la connaissance intime des choses qui, grâce à l'examen des documents présentés et au recours à des témoignages, permettent de trancher immédiatement, mais aussi la simplicité des comptes et leur lisibilité.

\section{La vie religieuse}

En distinguant les offrandes reçues à l'église Saint-Guénolé de celles qui l'ont été à Notre-Dame-du-Mûrier, le compte permet de préciser le rapport entre les deux églises. Pour la période du 22 mars 1478 (jour de Pâques) au 14 mars 1479, les offrandes provenant de Saint-Guénolé sont plus nombreuses (73 occurrences, contre 32) et le total récolté est plus élevé (35 l $2 \mathrm{~s} 6 \mathrm{~d}$, contre $18 \mathrm{l} 13 \mathrm{~s} 1 \mathrm{~d}$ ). Saint-Guénolé est l'église principale, celle où a lieu la grand-messe du dimanche, et la succession régulière des offrandes des dimanches se suit du 24 mai 1478 au 14 mars 1479. Cependant, pour la période du 29 mars au 3 mai, et pour le 11 mai les offrandes du dimanche ne sont recueillies qu'à Notre-Dame-du-Mûrier. Impossible de préciser s'il s'agit là d'une situation habituelle ou momentanée qui pourrait être liée aux travaux réalisés dans l'église Saint-Guénolé.

Les cinquante-deux dimanches qu'enregistre le compte représentent un apport de 26 l 14 s 2 d (70,5 \% provenant de Saint-Guénolé). Lors les dimanches " ordinaires " (c'est-à-dire sans comprendre ceux de Pâques, de Pentecôte, de la Trinité, de la Fête-Dieu, ni le jour de Toussaint qui, cette 
année-là, est un dimanche, non plus que le 31 mai, jour où la procession de Guérande est à Saint-Guénolé, et le 5 avril où certains font leurs Pâques), le montant des offrandes est variable, se situant entre $21 \mathrm{~d}$ le 3 mai, et $7 \mathrm{~s}$ $5 \mathrm{~d}$ le 24 octobre; le montant moyen s'établissant à $4 \mathrm{~s} 7 \mathrm{~d}$. Après les mois de mars et de mai riches de fêtes liturgiques, les dons de juin à fin septembre se situent au-dessous de la moyenne. Celle-ci n'est dépassée que le 18 juillet, le $1^{\text {er }}$ août et le 5 septembre. À partir du 26 septembre, les dons sont proches ou au-dessus de cette valeur moyenne sauf exception (29 novembre, 31 janvier, 7 mars).

Les offrandes faites à l'église Notre-Dame-du-Mûrier doublent celles faites à Saint-Guénolé lors de fêtes religieuses du calendrier liturgique : Pâques et ses féries, Saint-Guénolé (27 avril), Ascension, Pentecôte et ses féries, Fête-Dieu, Assomption, Nativité de la Vierge, Toussaint et jour des morts, Présentation de Notre-Dame, Conception Notre-Dame, Noël, SaintÉtienne, Saint-Jean, le jour des Innocents, Circoncision Notre-Seigneur, Épiphanie, Purification Notre-Dame. Mais l'église Saint-Guénolé paraît seule le 22 avril, le 19 mai (Saint-Yves), le 24 juin (Nativité de saint Jean-Baptiste), le jour de la dédicace de l'église, et celui de la fête de saint Guénolé en mars; alors qu'à l'inverse les dons ne proviennent que de Notre-Dame-du-Mûrier lors de la fête de la Trinité (17 juin), de celle de saint Yves en octobre, et lors de la venue de Madame de La Roche, Françoise de Dinan, épouse de Guy XIV de Laval, seigneur de la Roche-Bernard et de Châteaubriant.

L'importance de Pâques et de ses féries (24\% des offrandes totales) est à souligner, ainsi que le cycle des fêtes liées au culte de la Vierge $(24,1 \%)$. Celui-ci voit se succéder la célébration dans les deux églises de l'Annonciation (25 mars, 6,7 \%, cette fête est également celle de NotreDame-du-Mûrier), de l'Assomption (15 août, 4,1 \%), de la Nativité NotreDame (8 septembre, 3,9\%), de la Présentation Notre-Dame (21 septembre, $0,8 \%$ ), de la Conception Notre-Dame (7 décembre, $2,3 \%$ ), de la Purification (2 février, 4,8 \% compris la vente de chandelles). Il n'est que la Visitation (2 juillet) qui n'apparaît pas, sans doute faut-il penser pour ce jour à un pèlerinage attesté par la suite. Le lien entre l'église Notre-Dame-du-Mûrier et le culte marial est fort. Il est lié à l'origine même de la chapelle - la dévotion à la Vierge ayant conduit à sa reconstruction, et la demande d'indulgences qu'obtiendraient les fidèles la visitant est satisfaite lors des fêtes de la Vierge ${ }^{18}$. Le lieu a une certaine attractivité, une procession vient de Guérande le dimanche qui suit la fête du Sacre - le dernier dimanche de mai -, et de la Roche-Bernard vient Françoise de Dinan.

Les autres fêtes du calendrier liturgique apparaissent : la Pentecôte et ses féries des lundi et mardi (5,5\% des offrandes totales); la Toussaint et le jour des morts (5,7 \%); Noël (3\%); la Fête-Dieu (2\%); la Circoncision NotreSeigneur ( $1^{\mathrm{er}}$ janvier, 1,4\%), l'Épiphanie (6 janvier, 1,2 \%). Pour l'Ascension, la Trinité, la naissance de saint Jean-Baptiste, la Transfiguration, la Saint-

18. BourdeAut, A., La chapelle..., op. cit., p. 20. 
Étienne (26 décembre), la Saint-Jean (27 décembre), la fête des Innocents (28 décembre) les offrandes ne représentent pour chacune de ces fêtes que $1 \%$ ou moins des offrandes totales.

Les fêtes associées aux saints d'origine bretonne sont à relever : saint Yves, en mai et en octobre (1,5\% des offrandes totales); saint Guénolé qui est le patron de l'église paroissiale, fêté en avril et en mars, et lors de la dédicace de l'église (4,5\%).

La période du Carême et la semaine de la Passion sont des moments importants d'édification. Il est alors fait appel à un " professionnel de la parole ", frère François Dignan, dominicain du couvent Saint-Yves de Guérande, pour prêcher tout le long du Carême de la Passion et de la Résurrection. Une somme d'argent, une aumone, selon le religieux, lui est versée. La quittance qu'il rend de cette somme est datée du 6 juillet 1478, c'est-à-dire à une date assez éloignée de la fin du Carême. Les nobles, bourgeois, et gens notables de la paroisse du Batz, c'est-à-dire les éléments représentatifs de la paroisse, sans doute le général de la paroisse, s'expriment dans une autre pièce, également du 6 juillet 1478. Ils justifient la dépense engagée, en se référant à une précédente décision, et soulignent que la somme versée n'est qu'une compensation à l'absence de quête en faveur du couvent causée par la présence à Batz d'un des religieux. Les interventions dans la marge du contrôleur révèlent des tensions : la dépense est pour cette fois seulement; elle ne devra pas à l'avenir être supérieure. Surtout, une main a porté que dempuis la visitation du Croisic de Monseigneur de Nantes, il est défendu d'engager l'argent de la fabrique pour la venue d'un prédicateur. Épisode des relations parfois difficiles entre ordres mendiants et hiérarchie séculière ${ }^{19}$ ? Ou plutôt simple réaction face à la procédure employée? Frère François Dignan évoque comme commanditaire de sa venue Messeurs les bourgoys du Croisic, ce qui paraît être une allusion à l'institution municipale qui s'est mise en place au Croisic dans les années $1460^{20}$. Institution qui, ici comme ailleurs, intervient dans ce domaine et laisse à la fabrique une partie des frais occasionnés par la venue du prédicateur, le reliquat de ce que produit la quête n'ayant pas permis de solder. La mention de la venue de l'évêque de Nantes au Croisic, et non à Batz, peut encore donner corps à cette seconde hypothèse. L'évêque affirme ainsi le principe d'autonomie de la fabrique par rapport à d'autres instances laïques de décision.

Le souci du culte et de sa solennité se retrouve dans la présence d'un orgue et d'un organiste pour lequel il est dépensé une somme non négligeable : $10 \mathrm{l}$ par an. La présence d'une tapisserie, les travaux entrepris à l'édifice, l'achat d'objets cultuels et de vêtements sacerdotaux, le contrôle pointilleux des fonds de la fabrique illustrent encore la prise en compte de

19. MARTIN, Hervé, Les ordres mendiants en Bretagne, vers 1230-vers 1530, Paris, 1975; id., le métier de prédicateur en France septentrionale à la fin du Moyen Âge (1350-1520), Paris, 1988

20. Gallicé, Alain, Guérande..., op. cit., p. 194-199. 
" l'intérêt général ". Nul doute que l'ampleur des travaux réalisés exprime encore une identité locale et son affirmation.

Le document renseigne peu sur l'intérieur de l'église Saint-Guénolé et les dévotions dont elle est le cadre. Il est cité une chapelle Notre-Dame, sans doute celle de Notre-Dame-de-la-Clarté. D'autres documents font état en l'église Saint-Guénolé des chapellenies Saint-Guillaume, Saint-Louis, du Saint-Sacrement, Saint-Yves, de la Trinité fondée par Jean Le Pennec, seigneur de Kerdour en Batz et père de Michel Le Pennec, alors qu'une chapellenie Notre-Dame est mentionnée en l'église Notre-Dame-du-Mûrier ${ }^{21}$.

La déclaration des objets cultuels apporte quelques informations sur les images religieuses. Nous avons déjà présenté la grande croix de procession où figurent les Évangélistes, le crucifix, les Docteurs de l'Église, un Agnus Dei et une garniture décorée de fleurs de lys. L'iconographie religieuse se complète de représentations de saint Guénolé que l'on trouve sur une des tasses, sur une des croix, décorée également de fleurs de lys, et encore par deux fois associé à un crucifix sur un calice œuvré jusqu'à sa coupe. Un des calices présente au haut de la patte les Évangélistes. Le pommeau d'un autre calice est œuvré et, en sa patte, il y a un crucifix d'émail. Aux symboles de l'Église universelle, crucifix, Évangélistes, Docteurs de l'Église s'ajoute la dévotion au patron de la paroisse : saint Guénolé. Quant aux fleurs de lys, elles ont ici leur signification de blancheur immaculée qui renvoie au culte marial.

L'importance des reliques est à souligner : la chapelle présente des suaires en reliquaires; un tableau d'argent en contient; en une teste d'argent se trouve un reliquaire de saint Antoine; une petite tourelle en manière de chandelier renferme encore des reliques; et il est indiqué une autre petite croix où y a de la Vraye Crouex comme en un pommeau d'argent doré.

Pour deux des objets cultuels, l'origine est indiquée : une tasse porte le nom du donateur, le prêtre Jean Cario; une autre a été offerte par dom Bonabès Enes. Ce dernier nom se retrouve dans un autre compte de la fabrique de Batz tenu du 5 mars 1575 au 24 mars 1576, où un Bonabès Enes figure comme légataire d'un bréviaire et d'une légende, sans doute un exemplaire de La légende dorée de Jacques de Voragine. Ce document révèle encore les noms de généreux donateurs de vêtements sacerdotaux, décédés ou encore en vie en $1479^{22}$.

21. Arch. dép. de Loire-Atlantique, G 304, pour 1511; G 304, 3 juin 1478; B 1489 (A), $\mathrm{f}^{\circ} 64$; B 1489 (B), $\mathrm{f}^{\circ} 72 ; 1$ E $259, \mathrm{f}^{\circ} 3 ; \mathrm{H} 603, \mathrm{f}^{\circ} 31 ; \mathrm{B} 1489$ (A), $\mathrm{f}^{\circ} 65 ;$ B 1489 (B), $\mathrm{f}^{\circ} 73 \mathrm{v}^{\circ}$; B 1489 (A), $\mathrm{f}^{\circ} 99 \mathrm{v}^{\circ}$; B 751, 9 février 1469 ; B 750 et G 383/1, 5 août 1480 .

22 Arch. dép. de Loire-Atlantique, $1 \mathrm{~J} 134, \mathrm{f}^{\circ} 1 \mathrm{v}^{\circ}-2$. L'absence de dates, l'usage des mêmes prénoms sur plusieurs générations rendent difficile l'attribution à tel ou tel cité dans le compte. Relevons les noms de Jean André, Jacques, Jean et Guillaume Jouan, Guillaume Le Boseuc, dom Guillaume Le Bourdiec, Jean Le Calloch, Michel Le Pennec, Éon Maro, Jean Terruelle. 
Les deux églises sont des lieux d'inhumation. À Saint-Guénolé et à NotreDame-du-Mûrier, il en coûte $40 \mathrm{~s}$ pour être inhumés en cuer et $20 \mathrm{~s}$ hors le cuer $^{23}$.

Le contrôle des procureurs révèle une autre réalité religieuse. Ce contrôle est d'abord comptable, il est exercé par le commissaire nommé par l'autorité religieuse, sans doute le vicaire de Batz, Nicolas le Goyc, et des paroissiens présents. Il se manifeste par des annotations en marge qui accompagnent chaque paragraphe. Elles expriment le plus souvent une acceptation justifiée par une confrontation à des pièces comptables ou à des rapports identifiés par les noms de leurs auteurs ou de leurs signataires, ou encore à des précédents faisant référence, et parfois encore à la relation des assistants. Elles sont parfois plus bavardes lorsqu'il s'agit de préciser une règle nouvelle imposée par la hiérarchie, ainsi pour la prise en compte comptable de la lettre de procuration. Le contrôle ne se limite pas à entériner les articles enregistrés. La somme demandée pour la lettre de procuration est réduite et quelques paragraphes sont barrés et assortis en marge de la mention alibi (pour signifier que l'article est inscrit par ailleurs). Plus largement, la forme du compte, la procédure de son examen, le fait que les nouveaux procureurs doivent retirer leur lettre de procuration à Nantes sont les signes d'une forte présence de la hiérarchie religieuse qui se manifeste encore par la visite de l'évêque, visite pastorale que l'on prépare, qui a un coût et peut déboucher sur des décisions immédiatement applicables comme le montre la prise en charge des frais liés à la présence d'un prédicateur.

Le contrôle porte encore sur les procédures préludant aux décisions, à la désignation de l'exécutant, au paiement des travaux. Il est exercé alors par la communauté paroissiale ou plutôt ses représentants. Ainsi, le compte éclaire-t-il des aspects de la vie de la paroisse.

\section{Démographie, vie sociale et économique}

D'un point de vue démographique, il est difficile de tirer parti des quelques indications chiffrées. Que peuvent représenter les 301 écuelles qui contribuent au denier de Pâques? Ce chiffre renvoie-t-il à des unités fiscales ou à des foyers réels? La liste a-elle été actualisée?

Plus intéressant est l'examen de la liste des inhumations. Il en est recensé 49 . C'est relativement peu ${ }^{24}$, pour une paroisse peuplée de 4000 habitants ou plus. Sans doute faut-il envisager un sous enregistrement, en particulier des nouveaux-nés. On dénombre 24 adultes et 25 fils ou filles - ces mots fils et filles sont délicats à interpréter, mais ils doivent renvoyer à des mineurs. Une certaine surmortalité masculine apparaît,

23. Les Bretons et Dieu. Atlas d'histoire religieuse, 1300-1800, coord. CROIx, Alain, Rennes, carte 21 .

24. Il en est enregistré 135 entre le 25 mars 1575 et le 24 mars 1576, Arch. dép. de LoireAtlantique, $1 \mathrm{~J} 134$. 
27 individus contre 22 de sexe féminin, elle concerne moins les adultes (12 décédés et 12 décédées dont 4 sont des veuves) que les jeunes (15 fils, et 10 filles). D'autre part, la mort frappe peu à l'intérieur d'une même famille; cependant Jean Le Botec et Jean le Sénéchal perdent chacun deux fils, alors que Jean Porsal voit disparaître un fils et une fille et que Thomas Le Sénéchal et son épouse décèdent dans l'année. Apparemment, la situation démographique n'a rien de catastrophique. Après la crise, Batz figure dans une liste de rabais de fouage datée du 3 mars 1473 et son dégrèvement porte sur la totalité des feux ${ }^{25}$; plus largement, après la conjoncture déprimée des années 1472/1477, la pression de la mortalité est moins forte et sans doute Batz connaît-elle une phase de reprise.

Onze inhumations, $22 \%$, se font dans le chœur d'une ou de l'autre église. Elles concernent six femmes : quatre épouses, celles de Thomas Le Sénéchal, de Guillaume Houat, de Mahé Foenant, de Valentin Pierre et deux veuves celles de Rodrigo de Guerrande, de Guillo Crauzon; quatre hommes : dom Guillaume de Henleix, Jean Michel, Thomas Le Sénéchal et Jean Le Huedez; et un fils, celui de Jean Quelo. Ces sépultures illustrent des hiérarchies sociales et des possibilités financières.

Les hiérarchies sociales se devinent encore dans la dénomination de certains des décédés pour lesquels il n'est pas fait mention de prénom : la fille au Gloanec, la femme au Medec et surtout dans la liste rédigée concernant le devoir de la robe des morts. Il n'en est enregistré que seize (32\% des inhumés). Les procureurs ayant soin de déclarer que les autres n'avaient qu'une robe et étaient pauvres. Les choses sont sans doute moins simples, quatre de ceux qui ne contribuent pas sont inhumés dans le chœur d'une des églises : la veuve Rodrigo, la femme de Mahé Foenant, dom Guillaume Henleix, et le fils Jean Quelo. Certes, on peut penser que le fils n'avait encore qu'une robe (mais, la liste mentionne les robes de la fille de Jouhan Jac, et du fils de Jean Mahé), que pour le prêtre les règles sont particulières, mais on peut tout autant songer à quelque accord passé ce que suggère l'exemple d'Éon Moro qui laisse à la fabrique $1 \mathrm{l} 10 \mathrm{~s}$ mais ne cotise pas au titre de la robe des morts. La valeur moyenne des robes prélevées s'établit à $8 \mathrm{~s} 8 \mathrm{~d}$, les valeurs déclarées se situent entre $18 \mathrm{~s} 4 \mathrm{~d}$ et $2 \mathrm{~s}$, soit un écart de 1 à 9 . Sept valeurs se situent au-dessus de la moyenne, celles des robes : de la femme de Valentin Pierre, 18 s 4 d; de Thomas Le Sénéchal, 16 s 8 d; de la veuve Guillo Crauzon, $15 \mathrm{~s}$; de Louis Le Gannat, $10 \mathrm{~s}$; de la femme Le Sénéchal, $10 \mathrm{~s}$; de la femme Guillaume Houat, $10 \mathrm{~s}$; du fils Jean Mahé, $10 \mathrm{~s}$. Au contraire, neuf sont au-dessous de cette moyenne, la valeur la plus basse étant $2 \mathrm{~s}$ pour Jean Le Huedez. Certaines valeurs interviennent à plusieurs reprises, $10 \mathrm{~s}$, par quatre fois, $7 \mathrm{~s} 6 \mathrm{~d}$, par quatre fois, et $5 \mathrm{~s}$ à deux reprises. Les robes de femmes sont plus nombreuses, huit occurrences (sept de

25. Arch. dép. de Loire-Atlantique, $B \quad 7, \mathrm{f}^{\circ} 41$. Les fermiers de la recette des ports et havres de la comté de Nantes et de la recette ordinaire de Guérande depuis le $1^{\text {er }}$ octobre 1470 obtiennent un rabais important en raison des pertes subies, GalLICÉ, Alain, Guérande..., op. cit., p. 218 
femmes et d'une veuve, de $18 \mathrm{~s} 4 \mathrm{~d}$ à $4 \mathrm{~s} 2 \mathrm{~d}$, moyenne $9 \mathrm{~s} 8 \mathrm{~d}$ ); contre six pour les hommes (de $16 \mathrm{~s} 8 \mathrm{~d}$ à $2 \mathrm{~s}$, moyenne $7 \mathrm{~s} 10 \mathrm{~d}$ ), et 2 de fils et fille (de $5 \mathrm{~s}$ à $10 \mathrm{~s}$ ). Cette liste comporte une famille noble, Quelo. Mais, figurent surtout les noms de familles de marchands mariniers croisicais - Crauzon, Goezval, Houat, Jac, Le Huédé, Le Medec, Le Sénéchal, Mahé, Terroualle, Rodrigo - illustrant la position sociale occupée par cette catégorie ${ }^{26}$.

D'un point de vue plus général, le document éclaire le fonctionnement de la fabrique. On ignore le mode de nomination des procureurs. Toute décision implique l'avis, la présence et le commandement de paroissiens dont les noms sont cités - par exemple Jean André, Denis Quelo, Jacques Jouan, Jean Conan, Le Boteuc pour le marché des deux lucarnes -, souvent accompagnés de l'habituelle mention de plusseurs autres. Le processus décisionnel n'apparaît pas, sans doute y a-t-il accord de quelques-uns, des nobles, bourgeois et gens notables de la paroisse, que l'on peut assimiler au général de la paroisse et l'énoncé des décisions prises au prône de la grand-messe n'est là que pour faire passer une information aux paroissiens ${ }^{27}$. Si des travaux sont engagés un feur ou devis est établi, des bannies sont criées lors la grandmesse, une adjudication a lieu, le contrôle des journées de travail est assuré si nécessaire, un renable est établi, des quittances sont rédigées et certaines sont passées devant la cour de Guérande, par exemple celles concernant le versement du salaire de l'organiste, les paiements faits à Silvestre Guyomarc'h, de Rhuys, et à Olivier Thefoche, de Pénerf, ou encore au charpentier Jean Jarno. Toute vente par la fabrique fait l'objet d'une procédure au cours de laquelle se succèdent : une annonce au prône de la grand-messe, des bannies, une mise aux enchères; ainsi en est-il pour la vente de vieilles ardoises annoncée par trois fois lors de la grand-messe de trois dimanches successifs, alors que trois enchères font monter le prix de $50 \mathrm{~s}$ à $60 \mathrm{~s}$, Rio Talic étant le dernier bouteur. Le respect des procédures est gage de l'intérêt général et assure une relation de " proximité " avec les paroissiens.

Les procureurs exécutent les décisions prises, suivent les travaux. Pour les achats de certains matériaux, l'un d'eux se déplace et passe marché. Il peut s'adjoindre un spécialiste comme à l'occasion de l'achat des ardoises ou de clous. Leur rôle de gestionnaire se retrouve dans l'organisation des adjudications et dans les nouvelles baillées de rentes - deux cas sont signalés dont l'un a permis de porter la rente levée de $5 \mathrm{~s}$ à $8 \mathrm{~s}$. Le compte illustre encore le rôle de comptables qui est le leur. Les demandes d'apurement en fin d'exercice attestent qu'ils sont responsables des sommes maniées. En cela, ils sont mandataires de leur communauté, ou plus précisément du général, responsable devant celle-ci, la fabrique disposant d'une personne morale. Ainsi, les fabriqueurs sont-ils bien les procureurs de la paroisse, mais leur marge de décision est limitée ${ }^{28}$.

26. GALLICÉ, Alain, "Les marchands mariniers... ", op. cit., p. 174-186.

27. Raison du Cleuziou, A., "Trois actes prônaux. XV'-xvI ${ }^{\mathrm{e}}$ siècles ", Revue de Bretagne, 1905 , p. $398-405$; p. $66-80$.

28. Sur ce mot, RigAUDIÈRE, Albert, " Les procureurs urbains en Auvergne, Velay et 
Concrètement, le corps paroissial est représenté par un certain nombre de noms cités souvent à plusieurs reprises comme témoins des actes passés. Si la mention des noms au bas d'une quittance d'abord vise à donner crédit à l'acte, il n'en demeure pas moins qu'ils sont retenus en raison de leur caractère de notable. Aussi, le relevé permet-il d'esquisser une liste de notabilités constituée des nobles, bourgeois et gens notables de la paroisse du Batz. Cette désignation traduit une forte différenciation sociale et la conscience que cette oligarchie a d'être, selon la formule consacrée alors, la plus maire et plus saine partie de la population, l'administration paroissiale confirmant et renforçant la hiérarchie sociale. Dans la liste des témoins laïcs figurent : Jean André, Jean Bouchart, Tanneguy Chalcot, Jean Conan, Bernard Flahart, Guillaume Goury (ou Gouric), Jacques Jouan, Jean Jouan, Guillaume Jouan, lieutenant, Hervé Layc, Guillaume Le Boseuc, Jean Le Boteuc, Jean Le Bourdiec, Jean Le Gliff, Mahé Le Gliff, Michel Le Pennec, seigneur de Lauvergnac, Simon Le Rouxeau, Denis Le Terruelle, Éon Le Texier, Jean Mahé, Guillaume Marcraye, Jean Maro, Denis Quelo, Olivier Rio, Pierre Rogon, Olivier Ylisploé. Soit vingt-six noms, la liste n'étant sans doute pas exhaustive puisque la mention et plusieurs autres est utilisée à maintes reprises. Des nobles y figurent : Jean André, Jean Bouchart, Jean Conan, Jean Le Boteuc, Michel le Pennec, Simon Le Rouxeau, Pierre Rogon; ainsi que des anoblis, les Jouan, Guillaume Le Boseuc, les Le Gliff, Denis Quelo et un exempt de fouage, Hervé Layc, ces derniers ayant été distingués par le pouvoir ducal en raison des services militaires maritimes de leurs parents. Ils sont pour la plupart liés au Croisic, et ils ont gardé un lien avec l'activité maritime; on retrouve des représentants de ces familles servant sur mer lors de la guerre d'Indépendance. Guillaume Jouan exerce des responsabilités militaires : il est lieutenant du capitaine du Croisic. D'autres sont des officiers de finances : Michel Le Pennec, Jean le Boseuc, Jean Le Boteuc, Jouan. D'autres encore exercent comme notaires : Jean André, Jean Conan, Jacques Jouan, Jean Le Boseuc, Pierre Rogon. À ces nobles et anoblis s'ajoutent d'autres personnes dont la plupart sont liées au Croisic : Tanneguy Chalcot, Bernard Flahart, Jean Le Bourdiec, Denis Le Terroualle, Jean Mahé, Guillaume Marcraye, Jean Maro, Olivier Ylisploé et sans doute Olivier Rio. Le monde des marchands mariniers en particulier est représenté par Tanneguy Chalcot, Bernard Flahart, Jean Le Bourdiec, Olivier Ylisploé, alors que Guillaume Goury, Guillaume Marcraye et Jean Maro sont des notaires ${ }^{29}$. Ainsi, apparaît l'importance de la ville du Croisic, des nobles, des officiers, mais la bourgeoisie avec ses notaires et ses marchands mariniers n'est pas absente.

Lyonnais aux XIV et Xve siècles ", Mélanges de l'école française de Rome, Moyen Âge, 114, 2002, p. 121-159.

29. GALLICÉ, Alain, « La noblesse dans le pays guérandais (vers 1400-vers 1540), Enquêtes et documents, 2003, p. 13-36; id., "Une trajectoire sociale : Michel Le Pennec (1452-1495) ", Bulletin de la Société archéologique et historique de Nantes et de la Loire-Atlantique, 2003, p. 95-118. 
La liste des témoins présents aux divers actes de la vie paroissiale révèle encore le nom de prêtres : Raoul Bellego, Guillaume Deniel, Guillaume Le Bourdiec, Nicolas Le Goyc, Guillaume Peneto ou Panneto, Guillaume Pierre, Michel Sellac. À ces noms, s'ajoutent des chapelains dont il est difficile de préciser s'ils sont des desservants de chapellenies dans la période du compte : Bonabès Enès, Pierre Hervé, Pierre Le Loues, Bernard Payen ${ }^{30}$. Cette liste sans aucun doute incomplète atteste un important clergé. Elle illustre encore l'origine locale d'une partie du bas clergé séculier. La présence des familles Enès, Le Bourdiec, Le Goyc, Sellac illustre la possibilité pour ces familles de marchands mariniers de donner un niveau d'étude suffisant à certains de leurs enfants, sans doute des cadets, pour accéder à ces postes.

Les trente-quatre rentes citées portent sur des biens répartis sur l'ensemble de la paroisse : maisons (huit, dont une à Kerdréan, une à Trégaté, deux au Croisic, dont une rue Neuve), masière (une au bourg de Batz), terres (à Caudan et Penchâteau), une vigne, un marais à Curusson, deux prés (près de Saint-Laurent et de la côte). Elles se situent entre 6 d et $30 \mathrm{~s}$. L'origine de ces rentes est rarement précisée. Nombre d'entre elles doivent être dues à des dons faits à la paroisse par testament, comme dans le cas de la veuve Jean Le Douille qui légua tous ses héritages et constitua ainsi une rente de $2 \mathrm{~s}$. Cependant, certaines rentes ont été constituées pour payer une inhumation effectuée - par exemple celle de Guillaume Yziquel - ou à venir. Dans ce dernier cas, il s'agit de prendre en charge l'enterrement d'une veuve soit par un versement échelonné - $5 \mathrm{~s}$ pour la veuve Éon Yziquel - ou par un versement en une fois dans le cas de la veuve Éonnet Herault, à effectuer par ses héritiers au décès de la veuve. Pour Julien Le Rouxeau et Geffroy Le Gruyer les rentes, respectivement de $24 \mathrm{~s}$ et $30 \mathrm{~s}$, portent sur l'ensemble de leurs biens par contrat de vente qu'il en a été fait à la fabrique. Pour ces deux personnages que l'on sait engagés dans les affaires commerciales maritimes, ces rentes expriment une réalité financière qui fait de la fabrique une sorte d'organisme de crédit en raison des disponibilités financières qui sont les siennes, traduisent une gestion des fonds possédés - la constitution d'une rente constituée peut être assimilée à un placement, mais elles sont encore la manifestation d'une solidarité envers certains paroissiens ${ }^{31}$.

La prise en compte de la levée de deux œillets de la saline Miroudrouc renseigne sur le mode d'exploitation : recours à un paludier, Jean Jego; sur le rendement : 2 muids et demi; sur le prix de vente du sel : 32 s 6 d le muid; et encore sur les éléments constitutifs du prix de revient : la part du paludier représente le quart du prix de vente; les frais de conduit : 2 s 6 d par

30. Supra, note 21.

31. Des fragments d'une liste de débirentiers de la fabrique pour la période du 24 février au $1^{\text {er }}$ avril 1479, Arch. dép. de Loire-Atlantique, $1 \mathrm{~J}$ 325/1-2. Certaines rentes ne figurent pas dans le compte de 1478/1479, en particulier 2 s versés par Éon Viollé, dit Courtebotte. S'agit-il d'une rente qui ne figurera que le compte suivant? 
muid; les frais d'entretien : 20 d par œillet; alors que 12 d sont versés de rente à la seigneurie de Campsillon de qui relèvent les œillets. Le revenu net s'établit à $25 \mathrm{~s} 1 \mathrm{~d}$ obole par œillet. L'autre œillet exploité dans la saline Scaffne ne rapporte que 22 s 10 d, ce qui illustre les différences de rendement qui existent d'une saline à une autre, réalité souvent méconnue. Une indication de métrologie est également fournie : un muid comprend 17 moets. Cette mention pourrait indiquer un changement récent de rapport entre l'unité de compte, le muid, et celle de mesure, le moet, sans que l'on puisse préciser le sens d'une évolution qui dans la baie de Bourgneuf est marquée, semble-t-il, par l'alourdissement de la charge qui passe de 25 sacs, en 1390, à 28 sacs, au début du XVI ${ }^{\mathrm{e}}$ siècle $^{32}$.

Les travaux entrepris apportent des informations sur le fonctionnement des chantiers. Les travaux de maçonnerie sous la gouttière de pierre se font du commandement de plusieurs gens notables, en leur présence, un feur est établi, et le marché est passé avec Nicolas Le Texier. Il s'engage à fournir la pierre, obtient 15 d pour chaque charretée de pierres rendue sur le lieu de travail, et il est payé par journée de travail. Outre le vin de marché $5 \mathrm{~s} 8 \mathrm{~d}$, les procureurs paient $2111 \mathrm{~s}$ pour les 41 charretées nécessaires, $9 \mathrm{l} 14 \mathrm{~s} 2 \mathrm{~d}$ pour la main d'œuvre (pour 37 journées de travail de maçons et 35 pour leurs serviteurs). Les maçons reçoivent l'aide de Guillaume Jean, febvre (forgeron), qui assure le pointage des marteaux facturé $113 \mathrm{~s} 9 \mathrm{~d}$. L'assemblage de la pierre exige de la chaux. Soixante-sept truellées, pour $55 \mathrm{~s}$, sont fournies par Silvestre Guyomarc'h, de Rhuys, la quittance de paiement est rendue le 4 mai 1478. La chaux est ensuite convoyée par mules de l'étier Sterbriec au Croisic, au bourg de Batz - il en coûte $8 \mathrm{~s}$-, puis destrampée, c'est-à-dire éteinte avec de l'eau et du sable, matériaux dont le transport est assuré par des femmes pour $4 \mathrm{~s} 2 \mathrm{~d}$. Ces opérations sont renouvelées pour 115 autres truellées, coût $5 \mathrm{l}$ (quittance du 28 juin), et $10 \mathrm{~s} 10 \mathrm{~d}$ sont accordés à Mahé Perrot envoyé à Pénerf acheter de la chaux parce que l'on craignait d'en manquer et que le chantier ne prenne du retard. Trente-deux autres truellées sont encore livrées au Croisic par Olivier Thefoche, pour $32 \mathrm{~s}$ (quittance 4 septembre), ce qui requiert à nouveau l'usage de trois mules et coûte $6 \mathrm{~s}$ de transport. L'emprunt d'une pipe de chaux au seigneur de Lauvergnac, Michel le Pennec, en attendant la livraison coûte $22 \mathrm{~d}$; alors qu'une autre commande de chaux passée à Georget Le Ray, de Pénerf, porte sur vingt-huit truellées et demi (un tiers selon la quittance), pour 28 s 6 d, et le transport de Sterbriec à Batz, $4 \mathrm{~s}$. Les quittances se suivent de mai à septembre, alors qu'il n'est compté que 37 journées de maçon. Les commandes successives de chaux donnent l'impression d'un chantier peu maîtrisé. Cela paraît tenir à la nature du chantier qui est passé à la journée. Les comptes sont rendus en confrontant registres de feur et marché, rapports du pointage des journées des travailleurs et renable. Dans ce programme de travaux, la main d'œuvre représente près de $43 \%$, la chaux $40 \%$, et les transports, $14 \%$.

32. BURON, Gildas, Bretagne des marais salants..., op. cit., p. 115; HocQUET, Jean-Claude, Le sel et le pouvoir. De l'an mil à la révolution, Paris, 1985, p. 478. 
Pour la charpente, Jean Jarno, le maître d'ouvrage retenu n'est présent que 15 journées sur les 45 de travaux, alors que Alain Pilet est présent trois journées et Éonnet Bonamy, une journée selon la quittance du 8 août. Ces charpentiers doivent constituer la compagnie de Jean Jarno, sans que l'on puisse préciser leur statut. Pour ces travaux, un prix ferme est donné, illustrant un autre mode de passation de marché.

Pour la couverture, 27 milliers d'ardoises d'Auvrigné (Auverné), et un millier et demi de lattes sont achetés à Nantes à Richebourg à Pierre du Tay, pour $53117 \mathrm{~s} 4 \mathrm{~d}$ versés le 22 août 1478 . L'un des procureurs, Jean Cabelguen, se déplace à Nantes accompagné de Guillaume Pihalle, un couvreur, pour une durée de six jours, il est compté $18 \mathrm{~s} 2 \mathrm{~d}$, et la location du bateau, $11 \mathrm{~s}$, une barge, qui les mène à Nantes, Guillaume Pihalle recevant en outre $5 \mathrm{~s}$ pour son salaire. La durée du déplacement s'explique entre autre par la visite à plusieurs fournisseurs et autant de négociations. Autre achat, celui de clous, deux sommes de clous lateretz et de tache acquises de Jean Ernaud, pour 4 l 12 s, et acheminées par mules, $2 \mathrm{~s}$, alors que le transport des ardoises et des lattes, à partir de Nantes, est effectué par trois navires et coûte $615 \mathrm{~s}$. Les navires utilisés sont des caboteurs présents à Nantes et disponibles pour des frets de retour. Ils sont d'Aber Ildut, d'Auray et de Piriac. Leur déchargement soit au Pouliguen soit au Croisic sur les quais coûte $11 \mathrm{~s} 1 \mathrm{~d}$; l'acheminement entre les ports et Batz engage $2112 \mathrm{~s} 9 \mathrm{~d}$, alors que $15 \mathrm{~s} 2 \mathrm{~d}$ sont encore alloués à Jean Cabelguen en passages et mises. Surtout la facture de couverture s'élève à $37 \mathrm{l} 10 \mathrm{~d}$ pour 254 journées d'intervention. Les contrôleurs indiquent en marge que le marché a été passé longtemps avant que les travaux ne commencent, ce que confirme l'absence d'indication de vin de marché. S'agit-il d'indiquer un retard pris par le chantier? Ou de se référer à un cahier des charges particulier et alors de justifier le coût des déplacements de Jean Cabelguen afin d'éviter que certaines dépenses ne soient remises en cause par les contrôleurs? Ou a-t-on eu recours à des spécialistes dont il faut réserver les services? Le fait que Guillaume Gourel se déplace spécialement pour indiquer la conduite à suivre aux charpentiers peut donner corps à cette dernière hypothèse. À ces clous et taches vient s'ajouter l'achat de chevilles, 14 milliers, et de clous tant de tillac que despuer, $32 \mathrm{~s}$ (quittance du 11 novembre 1478). Descendre et trier les pierres d'ardoise en coûte encore $12 \mathrm{~s}$, alors qu'est louée $30 \mathrm{~s}$, une maison proche de l'église, celle de dom Jean Johanno, pour y entreposer les ardoises, les bonnes étant stockées dans le cimetière. Il faut sans doute encore y ajouter que les frais de nettoyage de l'église et du cimetière lors de la venue de l'évêque, $2 \mathrm{~s}$, alors que $5 \mathrm{~s}$ sont consacrés à boucher une brèche apparue au cimetière, illustrant le soin, qu'ici comme ailleurs, on a à maintenir en bon état la clôture du cimetière. Au total, dans ce programme de travaux, la part des matériaux représente $54 \%$ des frais engagés, celle de la main d'œuvre, $33 \%$.

Un échafaudage est mis en place, avec quatre mats et cinq bourlingues venus du Croisic et tenus par des cordes de bouille, illustrant l'activité de construction navale de cette ville. Les matériaux sont hissés par une corde, 
et pour les plus lourds il est employé une haussière, cette dernière empruntée à Nicolas le Lessour.

La coordination des travaux est assurée par les professionnels euxmêmes. Ainsi, le couvreur Guillaume Gourel passe une journée, facturée 2 s $6 \mathrm{~d}$, pour advertir les charpentiers au commencement de leurs travaux. Il est également présent lors de l'achat de clous.

P.-A. Monnier a voulu voir dans ces travaux la réfection à neuf de la couverture et de la charpente de l'église, et la construction d'une voûte nouvelle en pierre de taille d'une longueur de 59 pieds et demi entre les deux nefs $^{33}$. De fait, le mot gouttière ne semble indiquer qu'un développement longitudinal; d'autre part la mention de travaux de menuiserie autour de la gouttière et de la chapelle Notre-Dame permettent de situer les travaux autour d'un bas-côté que l'abbé Bourdeault désigne comme celui de SaintFrançois ${ }^{34}$. L'expression entre les deux nefs sans plus de précision donne à penser à la finalisation d'une liaison entre un bas-côté et la nef ce qui entraîne une reprise en sous-œuvre de la main de la maçonnerie inférieure que l'on suit de mai à septembre. À cette date, les anciennes ardoises sont déposées et vendues. Puis la reprise des charpentes et de la couverture du bas-côté et d'une partie de la nef réalisées, on aménage des baies et on place des vitraux.

D'autres renseignements sont apportés sur l'origine des matériaux : la pierre employée au vu du coût du transport doit être d'origine locale, de la chaux vient de la péninsule de Rhuys, et de Pénerf; l'ardoise vient d'Auverné via le port nantais de Richebourg; pour lembruncher les lucarnes aux environs des noues, il est employé des planches de fou (hêtre) boys de Rouan et des planches provenant de fûts de pipe.

Le nom d'un certain nombre d'artisans est cité ainsi que des coûts et des salaires. Nicolas Le Texier et Guillaume Le Tilli appareillent de la pierre de taille entre les nefs, et prennent 9 s 7 d par pied posé. Nicolas Le Texier prend pour lui et ses despens $3 \mathrm{~s} 9 \mathrm{~d}$ par jour, les autres maçons reçoivent $3 \mathrm{~s}$, et leurs serviteurs, $2 \mathrm{~s} 4 \mathrm{~d}$. Le forgeron Guillaume Jean est présent sur le chantier et assure le pointage des marteaux. Jean Jarno est charpentier. Pour lui et ses despens, il perçoit $4 \mathrm{~s}$, les autres charpentiers de sa compagnie, $3 \mathrm{~s} 4 \mathrm{~d}$. Guillaume Pihalle et Pierre Gourel sont couvreurs. Aux oupvriers employés pour la couverture, il est accordé pour salaire et despans, $2 \mathrm{~s} 11 \mathrm{~d}$ par jour. Guillaume Le Peintre assure la réparation des vitraux, alors que Simon Uzel pose un vitrail à une lucarne. Alain Gaultier est menuisier, Éon Le Guiader fournit des verges et pièces métalliques pour les vitraux. Guillo Bretin assure la façon des étoffes. Une main d'œuvre féminine est recrutée sur ces chantiers. Elle est employée à porter de l'eau et du sable pour éteindre la chaux. Symon, Pezdron et Jacob Jouhanno assurent des charrois de même que Guillaume Alaire. Jean Gledel, marchand au Croisic,

33. MONNIER, Pierre-Aristide, Le pays guérandais..., op. cit., p. 210-211

34. BourdeaUt, A., La chapelle..., op. cit., p. 12. 
fournit des chevilles et des clous, mais aussi des tissus, il est en relation avec Rennes. Jean Cadoret vend des orseaulx, il est sans doute à rapprocher d'un orfèvre de Guérande. Jean Gego et Jean le Huedez sont paludiers. Le transport par charrettes ou par mules est pratiqué, des bateaux chargés de chaux venant de la péninsule de Rhuys arrivent dans l'étier Sterbriec où ils déchargent la marchandise transportée. Jean Ernaud qui vend des clous, sans doute au Croisic, et Pierre du Tay des ardoises et des lattes à Nantes signent leur quittance. Au-delà des renseignements sur le tissu économique local, ce sont des circuits économiques plus ou moins étendus et des mécanismes de circulation de l'argent qui apparaissent contribuant à une certaine régularisation sociale.

Des prix sont mentionnés : $5 \mathrm{~s}$ pour une trullée de froment, $10 \mathrm{~d}$ ou $10 \mathrm{~d}$ obole ou $12 \mathrm{~d}$ pour une truellée de chaux; $3 \mathrm{~s} 5 \mathrm{~d}$ pour un cent de lattes; $1 \mathrm{l} 18 \mathrm{~s} 6 \mathrm{~d}$ pour un millier d'ardoises d'Arverné rendu à Nantes; $5 \mathrm{l}$, pour un millier de clous lateretz (pour les lattes) et de tache; $7 \mathrm{~d}$, pour un millier de chevilles; $2 \mathrm{~s} 2 \mathrm{~d}$ pour un cent de clous de tillac, et $18 \mathrm{~d}$ pour la même quantité de clous de demi tillac; $18 \mathrm{~d}$, pour un cent de clous d'espeur; $10 \mathrm{~d}$, pour un cent de clous de chapeler; $5 \mathrm{~s}$, pour un pied de vitrail; $3 \mathrm{~s} 9 \mathrm{~d}$, pour une aune de bougrain; $7 \mathrm{~d}$, pour une once de fil noir.

Le compte de fabrique de la paroisse de Batz tenu du 22 mars 1478 au mars 1479 apporte son lot d'informations variées. Il illustre le fonctionnement de la fabrique qui agit par l'entremise de ses deux procureurs. Ceuxci sont les mandataires d'un groupe d'hommes qui se nomment les nobles, bourgeois et gens notables de la paroisse de Batz, assimilable au général de paroisse, dont l'administration épiscopale, qui contrôle de près la fabrique, paraît soucieuse d'assurer l'autonomie par rapport au pouvoir civil représenté par la municipalité croisicaise. Ce compte confirme encore la relative richesse du pays guérandais à la fin du $\mathrm{Xv}^{\mathrm{e}}$ siècle, l'affirmation de la ville du Croisic et de ses marchands mariniers. 


\section{Annexe}

\section{$[\ldots]^{35}$}

[folio 1]

Une chapelle d'argent en laquelle y a $[\ldots]$ et suaires en reliquaire sur quatre $t[\ldots]$ et $y$ a ung clochier ouquel y a deffault de deux croetz es deux boutz d'iceluy et sur le clochier y a deffault d'une crouez et guyrouette et pareillement sur deux desdites tourelles de deux guyrrouectes.

Une crouez à quatre euvangelistes d'ung costé doréz avecquez le cruxifi tout entier aient dyadesme et le tiltre et en l'autre costé les quatre docteurs de Saincte Eglise doréz et ung Agnus Dei doré avecques ung plommeau aient seix pans et en chacun pan ung ymaige d'argent doré sur lequel plommeau y a deffault en deux pignons de troys petites croez avecques et au pié de ladite crouez et au desus dudit plommeau en deux jointures de la fleur de lis dudit pié y a deffault de partie et de la garneture es deux bratz de chacun costé et au bout de hault d'une des fleurs de lis en deux lieux.

Une autre croez garnie d'argent ou y deffault, savoir es deux bratz d'icelle du bout $d[\ldots]$ au bout d'une des fleurs de lis et d'iceluy bout de l'autre costé par avant [...] environ et de l'autre bout du costé $d$ '[...] au petit bout de la fl[...] deffault et au bout de [...] et au-dessus sainct Michel.

[folio 1 verso]

[...] benoistier et esperiouer d'argent. Deux tasses d'argent chacune pesante ung marc ou environ en l'une des desquelles y a ung ymage de sainct Grimolay et en l'autre est escript Jehan Cario prebre.

Deux chandelliers d'argent esqueulx y a deffault de deux petiz rollets d'argent es piez d'iceulz.

Galices

Ung galice d'argent doré et esmaillé, la pate d'iceluy et le dehault de la pate à euvangelistes avecques sa plataine dorée.

Ung autre galice o sa plataine en la pate duquel y a ung cruxifi et deux ymages de sainct Grimolay esmaillez et au desus de ladite pate jucques à la coulpe oupvré.

Item neuff galices dorez o leurs plataines sauff une plataine qui n'est point dorée.

Galices d'argent

[...] differants galices d'argent et y a parties dorés o leurs plataines.

[folio 2]

Ung galice que a donné dom Benabes Enes, ledit galice veré.

Ung autre galice, le plommeau duquel est oupvré et en la pate d'icelui ung cruxifi d'esmaill et sa plataine.

Deux ensenciers d'argent o leurs chaynes et boucles.

Une pecine d'argent dorée en laquelley a deffault, savoir au desus de l'esmaill et partie des feillages.

Item ung tableau d'argent où y a plussieurs relicquez.

Item une teste d'argent où y a relicques de saint Anthoine.

Item y a en une poche plusieurs pieces d'argent cassé de demourant de la garneture d'argent des ymages, qui furent expletez.

Une petite croez en laquelle [...] quatre pierres es boutz d'icelle [...] au milieu.

35. Le document est incomplet. La transcription ne prend pas en compte les quittances insérées dans le compte. 
[folio 2 verso]

Item une autre croez petite où y a de la Vraye Crouez garnie d'un plommeau d'argent doré.

Item une petite tourelle en manière de chandelier où y a plusieurs relicques.

[écrit d'une autre main] Item deux petiz orseulz d'argent verez.

[folio 3]

Après comptent et se chargent lesdits procureurs du rest en quoy Jacquet Vinces et Guillo Cazdre precedans et derrains procureurs par avant lesdits lesdits Cabelguen et Jouhanno resterent pour la deducion du compte qu'ilz rendirent de leurdit office quel rest se monte la somme de. IIIIt $L X X L X I S \vee d$

[en marge] Apuré à la deducion du compte desdits Vinces et Guillo Cazdro.

Après comptent et se chargent lesdits procureurs des offeandes qu'ilz ont euz et receuz du tablier de ladite église monsieur saint Grimolay

Combien que l'office desdits procureurs commancza le $x X^{e}$ jour de mars qui fut le Vendredi benoit lesdits procureurs ne se chargent point de la recepte dudit jour par ce que les precedantz procureurs en firent la recepte et en ont compté.

[en marge] Il dit vroy.

le jour de Pasques.

LXVIII $s$ III $d$

le lundi de Pasques $X V I S$ V $d$

le mardi.....

$X$ XVIII $s$ II $d$

le mercredi qui fut le jour Nostre Damme ..........................................................III $s$ II $d$

Item les deniers qui furent levez [...] sur la table de cieulx qui recevoint le sacrement le jour de Pasques et les lundi, mardi et mercredi des feriers de ladite feste qui se monte .XVIII $s$ IX $d$

[en marge] le raport

[folio 3 verso]

le cinquiesme jour d'apvrill pour l'offrande de la table de ceulx qui firent leurs Pasques celui jour.

le XXII jour dudit moys pour l'offrande de ladite table ..............................................XII $d$ le jour Saint Grimolay en apvrill .......................................................................III $s$ IIII $d$

le jour de l'Ascension Nostre Seigneur.................................................................. $s$ II d

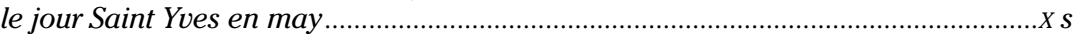

le jour de la Pentecouste ..................................................................................... $s$ X $d$

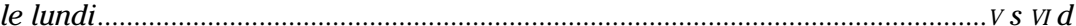

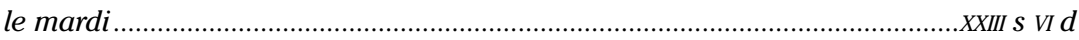

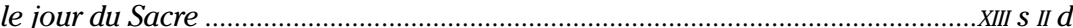

le dimanche que la procession de Guerrande fut en Baz.................................... V s I d

le jour de la Nativité saint Jehan Baptiste ........................................................... $s$ X d

le jour de la dedicacion de ladite eglise ................................................................... $s$ I d

le jour de la transfiguration Nostre Seigneur......................................................III $s$ II $d$

[en marge] comme davant

[folio 4]

le derroin dimanche de may que la procession de Guerrande fut en ladite eglise .....XXXVIII $s$ 


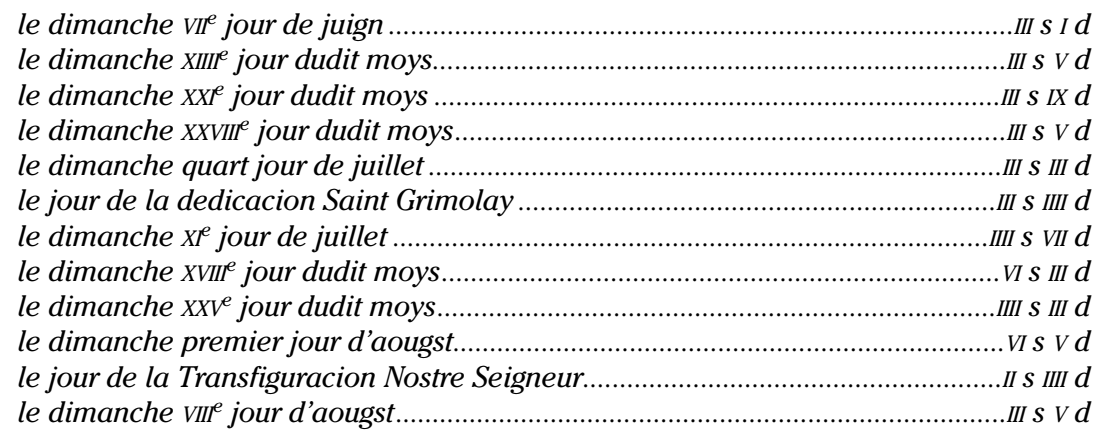

[en marge] comme davant

[folio 4 verso]

le dimanche quinziesme jour, jour de l'Asumpcion Nostre Damme.....

$X X X I X s$ VId

le dimanche XXII jour d'aougst.

le dimanche $X X I X^{e}$ jour dudit moys.

III $S$ IX $d$

le dimanche $v^{e}$ jour de septembre. V $s$ VIII $d$

le jour de la Nativité Nostre Damme.......................................................XXV S VII d

le dimanche XII jour dudit moys .......................................................................II $s$ I d

le dimanche $\mathrm{XIX}^{\mathrm{e}}$ jour dudit moys ...................................................................III $s$ XI $d$

le dimanche XXVe jour dudit moys ...............................................................................

le dimanche tiers jour d'octobre ........................................................................... S II d

le dimanche $x^{e}$ jour dudit moys ......................................................................... VI S III $d$

le dimanche XVIT jour..........................................................................III $s$ VI

le dimanche XXIIII jour dudit moys.................................................................. $s$ Vd

le premier dimanche de novembre qui fut le jour de Toussains........................XIX $s$ VII $d$

le jour des deffuncts ......................................................................................... VIII $s$ III $d$

le dimanche VIII jour de novembre ......................................................................IIII s III d

le dimanche $\mathrm{XV}^{\mathrm{e}}$ jour dudit moys....................................................................... $\mathrm{V} d$

le dimanche XXII jour dudit moys......................................................................IIII $s$ VII $d$

le jour de la Présentation Nostre Damme …………….......................................... VII $s$

[en marge] comme davant

[folio 5]

le dimanche XXVIII ${ }^{e}$ jour dudit moys............................................................................. $\mathrm{V} d$

le dimanche $v^{e}$ jour de decembre ................................................................IIII $s$ VII $d$

le jour de la Consepcion Nostre Damme...........................................................III $s$ IX $d$

le dimanche XII jour dudit moys.....................................................................IIII $s$ IX d

le dimanche $\mathrm{XIX}^{\mathrm{e}}$ jour................................................................................... $s$ VII $d$

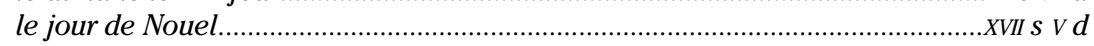

le dimanche XхVI jour dudit moys qu'estoit le jour Saint Estienne ............................ II $S$

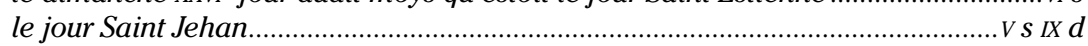

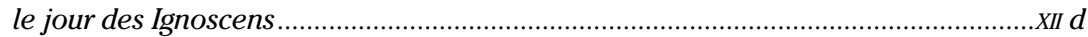

le jour de la Circunsicion Nostre Seigneur .............................................................. $s$ IX $d$

le dimanche second jour de janvier..............................................................IIII $s$ VII $d$

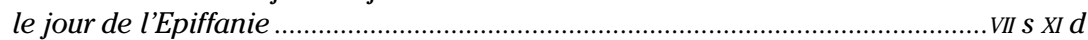

le dimanche $x^{e}$ jour dudit moys ........................................................................ $S$ V d

le dimanche XVII jour dudit moys ..................................................................III $s$ VIII $d$ 
[en marge] comme davant

[folio 5 verso]

le dimanche derroin jour de janvier...........................................................III $s$ V le jour de la chandeleur tant pour la chandelle que pour l'offrande dudit jour.......... $s$ VIII $d$ le dimanche vII jour de febvrier .....................................................................IIII $s$ III $d$ le dimanche XIIII jour dudit moys .................................................................IIII $s$ VII $d$ le dimanche $\mathrm{XX}^{e}$ jour dudit moys ....................................................................III $s$ Vd le dimanche derroin jour dudit moys.............................................................III $s$ VII $d$ le jour Saint Grimolay en mars........................................................................ $s$ III $d$

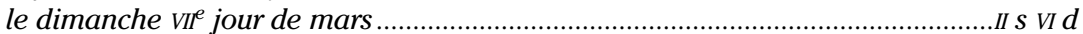
le dimanche XIIII jour dudit moys........................................................................ $s$ III $d$

[en marge] comme davant

[folio 6]

Après comptent et se chargent lesdits procureurs des offrandes du tablier Nostre Damme du Morier ainxin que la decleracion ensuit :

premier,

combien que l'ofice desdits procureurs commancza le Vendredi benoist $X X^{e}$ jour de mars, ilz ne comptent point les offrandes dudit jour pour ce que les precedantz procureurs en firent la recepte et en ont compté.

le jour de Pasques XxIr jour de mars ................................................................... $s$ Id le lundi de Pasques .................................................................................. $s$ IIII $d$

le mardi de Pasques ....................................................................................

le mercredi que fut la feste Nostre Damme .....................................................XXI $s$ IX $d$

le dimanche $x_{x I X}$ jour de mars ................................................................................... $s$ I

le dimanche $v^{e}$ jour d'apvrill ....................................................................... $s$ I d

le dimanche XII jour d'apvrill ............................................................................. VII $s$ III $d$

le dimanche $X I X^{X}$ jour dudit moys ....................................................................... VI $s$ II d

le jour dimanche XХVII jour et feste Saint Grimolay...............................................II $s$ III $d$

le jour de l'Ascencion Nostre Seigneur........................................................... $s$ V

le dimanche quart jour de may ...................................................................................

le jour de la Penthecouste qui fut Xf jour dudit moys........................................... $\mathrm{VII}$ III $d$

le lundi de la Penthecouste ..............................................................................................

le mardi de la Penthecouste................................................................................ $s$ VII $d$

le dimanche de la Trenité ......................................................................... $s$ III

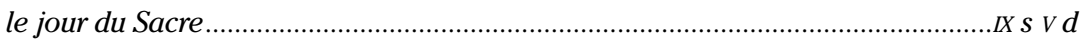

[en marge] o leur rapport

[folio 6 verso]

le jour que Madamme de La Roche fut à ladite église.................... $s$ III $d$

le jour de l'Asumpcion Nostre Damme............................................................... $s$ IIII $d$

le jour de la Nativité Nostre Damme ............................................................... $s$ VII

le jour Saint Yves en octobre............................................................................... $s$ V

le jour de Toussains .................................................................................... $s$ VI

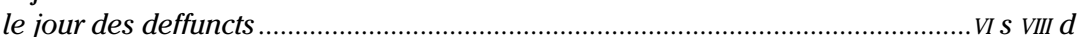

le jour de la Présentacion Nostre Damme ........................................................II $s$ VI

le jour de la Concepcion Nostre Damme .................................................................... $s$ 
le jour de Nouel............................................................................................... $\mathrm{S}$ II $\mathrm{d}$

le jour Saint Estienne ..............................................................................................

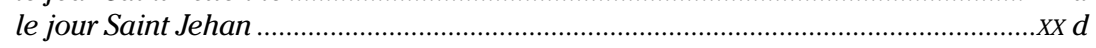

le jour des Ingnostens.....................................................................................................

le jour de la Circuncision Nostre Seigneur ....................................................... VI $s$ VIII $d$

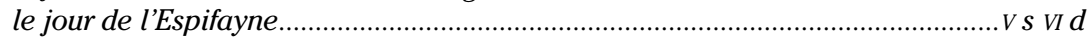

le jour de la Purificacion......................................................................................... $s$ X d

le jour de Saint Grimolay en mars .....................................................................

[en marge] comme davant

[folio 7]

Après comptent et se chargent lesdits procureurs du denier de Pasques que doivent les parroessiens de ladite parrouesse, savoir, cinq deniers par chacune escuelle qui ont esté receuz par la main dom Guillaume Le Bourdiec, secretan de ladite eglise, en oultre la somme de saixante soulz que ledit Bourdiec en a retenu pour son sallaire. Et est entendu que ledit debvoir est deu, savoir, de ancienne imposicion acoustumée III d par escuelle dont le prieur prent une maille. Quelle maille s'apelle maille de confession par chacune escuelle et de nouvelle imposicion est deu II $d$ par escuelle pour la sonnerie des orgues oultre lesdits III $d$. Ainsi demeure o ladite eglise, rabatu le droit dudit prieur, IIII d maille sur lesquelx le segretan prent d'ancienne ordonnance pour ses gages de servir ladite segretainerie II d maille par escuelle. Et par ce que sa charge est plus grande que de ancienneté lui avoit esté ordonné de nouveau pour la garde de la chappelle Nostre Damme du Morier LX s sur lesdits II d de nouvel imposez. Et est tenu par ce moien faire la cire de ladite eglise et de l'eglise Nostre Damme, faire lavanderies des ornemenz, fournir d'osties, et autres services requis environ lesdites eglises. Quel devoir se monte pour ledit an VI $L V S$ Vd

[folio 7 verso]

Après se chargent lesdits procureurs des deniers qui ont esté trouvéz es troncs et boestes de ladite eglise Nostre Damme du Morier qui furent oupvertz le XXIX jour de mars oudit an en la presence de dom Guillaume Peneto, dom Raoul Bellego, dom Michel Sellac, dom Guillaume Le Bourdiec, Jehan André, Jehan Le Botec et plusieurs autres, et y eust trouvé, comprins ce que fut trouvé en deux boestes de Saint Grimolay la somme de XXXIII L VI $s$ VII $d$, comme apiert par la relacion signée desdits hommes et pour ce .............XXIII $L$ VI $s$ VII $d$

[en marge] Appuré o la [...] relacion que [...] contient le contenu [...] ladite relacion [...] sur recepte desdits nommez.

Item le ve jour de decembre oudit an furent oupvertz lesdits troncs et boests Nostre Damme du Morier en la presence de Jehan Le Botec, Jean Conan, Guillo Layec, Olivier Ylisploé, Jehan Le Bourdiec, dom Raoul Belego, dom Guillaume Le Bourdiec, dom Guillaume Peneto, Denis Le Terielle et plusieurs autres comme apiert par la relacion de ce faite et $y$ fut trouvé dont se chargent lesdits procureurs la somme de. ..XXXVIII $L$ XVII $s[\ldots]$

[en marge] Apuré paraillement o sa relacion signée des contenuz en l'article qu'est demouré sur ce compte.

Item le ve jour de juillet oudit furent oupvertz lesdits troncs et boeestes Nostre Damme du Morier en la presence de Guillaume Jouan, de Guillaume Paneto et Raoul Bellego, dom Guillaume Le Bourdiec, dom Guillaume Deniel, Jehan Mahé et plusieurs autres, comme apiert par la relacion de ce faite et y fut trouvé dont se charge lesdits procureurs.. XVLXs[...] 
[en marge] Apuré paraillement o sa relation signée desdits nommez quelle est demeuree en ce compte.

\section{[...]}

[folio 8]

Item se chargent lesdits procureurs avoir eu et receu de Jehan Jouhan qu'il dona a ladite eglise

Item pour une trullée de fourment que dona Jehan Jouhan

Item se chargent avoir eu pour le paiement de quatre aulnes du bougrain de l'eglise pour l'enterrement et services de deffunct Eon Moro.................................................... $\mathrm{s}$

Item pour la licence de meptre et emporter la cire que furent es enterrement et service dudit dudit Moro

[en marge] o leur rapport.

Item pour le louage dudit an de deux estaux apartennant à ladite eglise [article barré et en marge est porté la mention alibi].

Item se chargent lesdits procureurs de la vente de la veille pierre d'ardayse de la despouille de ladite eglise venduz à Rio Talic par ban comme plus donant, en la presence de dom Guillaume Paneto, Jehan Conan, dom Raoul Bellego, pour la somme de ..IV L

[en marge] Apuré à la relation dudit acquest signé de Jehan Conan.

Item se chargent avoir receu de dom Jehan Le Maler pour une piecze de la veille goutiere de ladite eglise qui lui fut vendu pour le pris de. VII $s$ VI $d$

[en marge] o leur rapport et verificacion

[folio 8 verso]

Après comptent et se chargent lesdits procureurs des rentes à denier deues à ladite eglise par chacun an à prendre o les personnes qui ensuivent

premier,

Eon Sellac par Jouhan Sellac, son filz, la veuffve Perrot Caillaud, Georget Raoul, sur une meson o ses appartenances siis à Kerdrien. ...XXX $s$ Jamette Le Penfau, veuffve de deffunt Guillo Boulet............................................ 5 x $d$

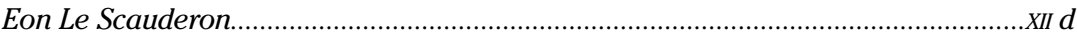

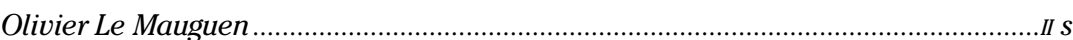

Guillaume Le Laguadec pour les filles Pezdron Laguadec........................................II s dom Guenolay Thebaud sur terres à Caudan que souloit autreffois tenir Jehan Le Pap, baillez audit Thebaud de nouvel ................................................................... $s$ VIII $d$ Jacob Jouhanno sur terres à Penchasteau que souloit autreffois tenir Jehan Le Pap et Pezdron Bourpaule ............................................................................................. $s$ III $d$ Morice Le Scauderon...............................................................................................IIII La veuffve Jehan Beaumenoir sur marois à Curusson...............................................II $s$ [article barré et en marge est porté la mention alibi] Jehan Even et sa femme pour l'enterrement Guillaume Yziquel .$X V d$

[en marge] selon les precedenz

[folio 9]

Guillaume Peneto sur une mazaire au bourg de Baz jouste la meson qui fut à Guillo Cazdre 
Jehan Guibourt en l'acquit de Denis Quelo qu'il eut [...] en l'acquit de Eon Yziquel et sa femme pour l'enterrement de ladite femme quant elle decebdera, sur une meson ou bourg de Baz davant la meson Olivier Le Laysour ...................................................... $s$ Jehan Le Guiolsec sur vigne ........................................................................................ $\mathrm{V}$ Jehan Vivant sur la meson que souloit tenir deffunt Jehan Regnaud à Kermabon, sur laquelle ledit Regnaud souloit poier $v$ s de rente qu'elle a esté baillée de nouvel par lesdits procureurs audit Vivant, à la somme de....................................................... VIII $s$

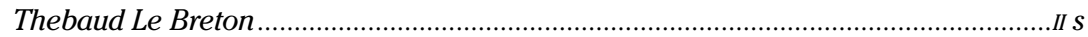

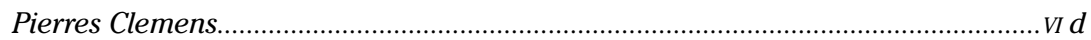

Jehan Landrain d'Escoublac ................................................................................... $d$

Simon Le Rouxeau sur la meson qui fut à deffunt dom Guyomarch Kerspern .. VII s VI d Jehan Heligan ................................................................................................ $\mathrm{x} d$

[en marge] comme davant

[folio 9 verso]

Jehan Le Bourdiec sur ung pré près Saint Lorans ............................................... VII s VI d

Jehan Talic de Kerderien.....................................................................................

Eonet Jouhan sur ung pré siis entre le pré Simon Le Rouxeau et la coste de Baz.... VII $s$ VI d Thomas Jouhanno sur sa meson à Tregaté et sur touz ses heritages par donaison de la veuffve Jehan Le Douille........................................................................................ $s$ Jehan Savaye et sa femme, fille Jehan Le Bedel .......................................................... $\mathrm{s}$ Perrot Le Botec...............................................................................................................III $s$ Jehan Le Speau par Philibert Le Fauer et sa femme, fille dudit Jehan Le Speau ....IIII $s$ VI d Jouhanette Le Bretonic comme heritiere de Jehan Le Bretonic sur sa meson jouste la meson Eon Foenant à Croesic............................................................................ $s$ Jehane Conan, veuffue de deffunt Jehan Beaumenoir sur marois à Curusson............II $s$ Guillaume Benoit en l'acquit de Rivel Le Roux ............................................................ VIII d

Nicolas Levevez et sa famme à cause d'elle ................................................................II $s$

[en marge] comme davant

[folio 10]

Katerine fille Mahé Huezlic $X V I d$

Les hers Eonet Herault sur une meson qui fut audit Herault en la rue Lenmer au Croesic...II $s$ Julien Le Rouxeau sur touz ses heritages par contrat de vente qu'il en a fait à la fabrique de la dite église. XXIV $S$ [...]

Geffroy Le Gruier sur touz ses heritages par contrat de vente qu'il en a fait à la fabrique

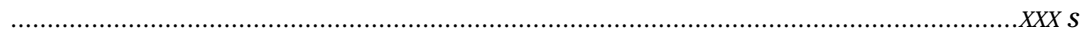

Guillaume Le Lan sur sa meson en la rue Neuffve qui fut autreffoiz à Jehan Toulin.......VI $s$

[en marge] comme davant

[folio 10 verso]

Après comptent et se chargent lesdits procureurs des enterrementz des corps qui ont esté enterrez en ladite eglise Saint Grimolay et en l'église Nostre Damme du Morier oudit an, savoir pour cieulx qui ont esté enterrez en cuers desdites eglises la somme de XL $s$ par chacun enterrement et hors les cuers de ladite eglise XX $s$ par chacun ainsi que ensuit :

premier,

pour l'enterrement du filz Jehan Le Seneschal 


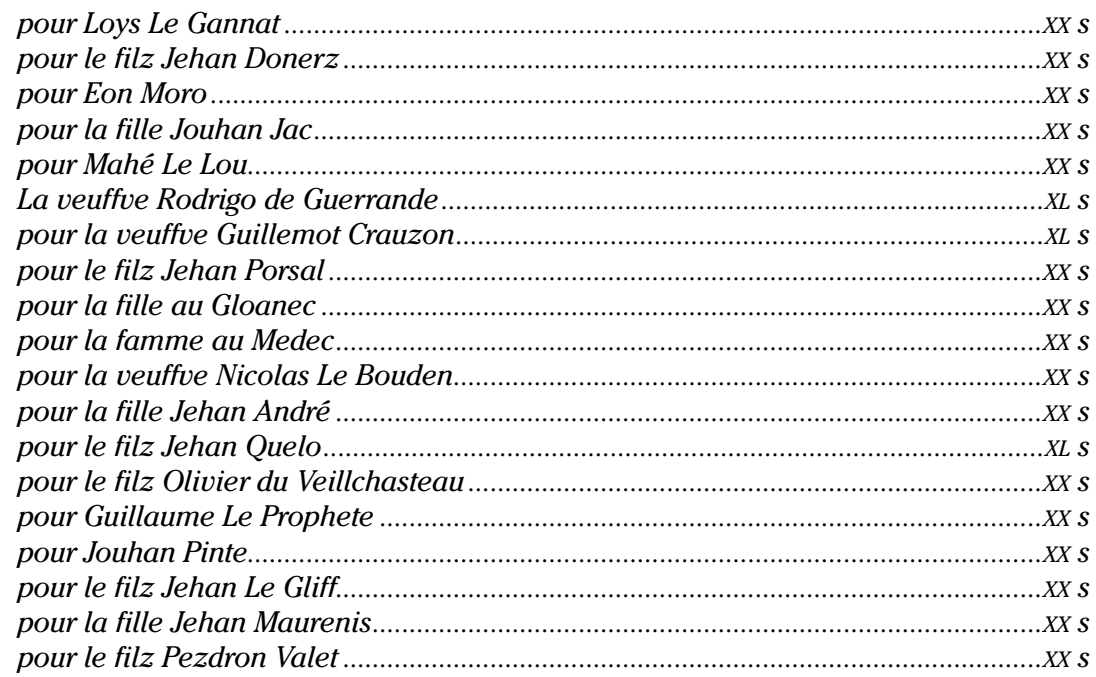

[en marge] Apuré o leur raport et à la relacion de dom Raoul Bellego comme quant à ce que aussi apiert par relacion signé dudit Bellego asistant [...] en la fin dudit escript.

[folio 11]

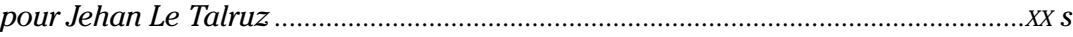

pour la famme Thomas Le Seneschal .......................................................................... $s$

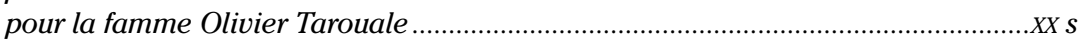

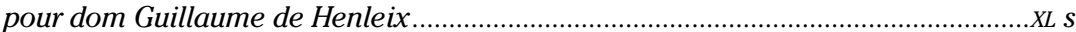

pour la fille Jehan Porsal.....................................................................................XX $S$

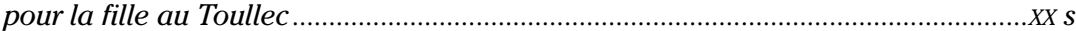

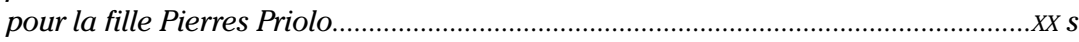

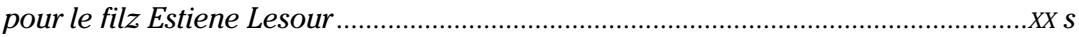

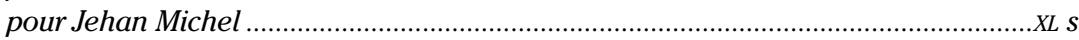

pour la famme Guillaume Houat....................................................................... $s$

pour le filz Eon Le Guiader......................................................................................... $\mathrm{s}$

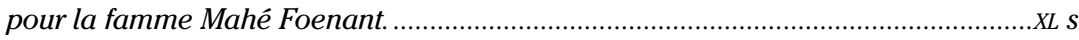

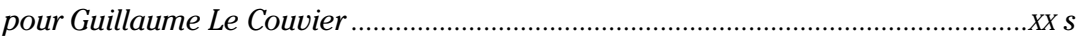

pour la famme Guillaume Cisaill..........................................................................XX s

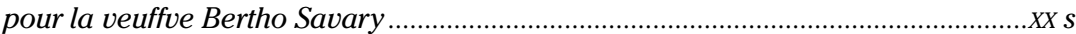

pour Jouhan Caiffas dit Go............................................................................XX S

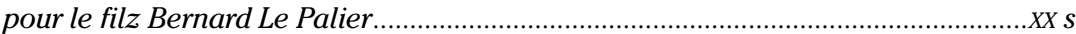

pour le filz Jehan Le Seneschal .........................................................................

pour le filz Geffroy Le Gruier................................................................................

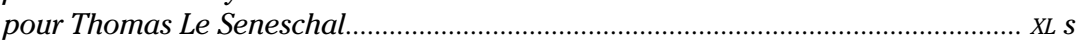

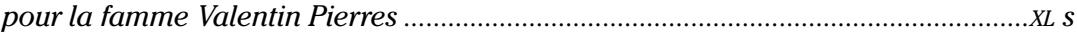

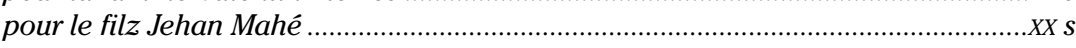

pour la famme Eon Goezval .............................................................................

[en marge] comme davant

[folio 11 verso]

pour Allain Conan 


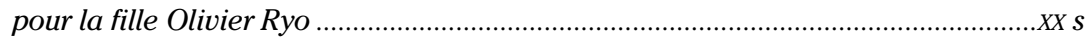

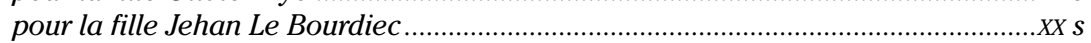

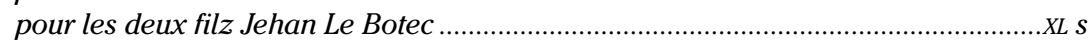

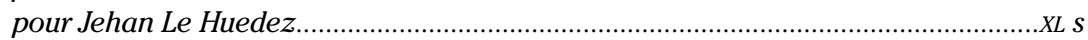
pour la fille Simon Le Rouxeau............................................................................ $s$ Raoul Bellego, prebre, commis des parroessiens de Baz quant affin de faire le raport des enterrrementz de ladite parroesse et d'en prendre les obligacions, certiffie le raport desdits enterrementz cy davant estre vroy et qu'il n'en y a plus large que soit venu à ma cognoessance, tesmoin mon signe manuel le XVIII jour d'apvrill l'an mil IIII ${ }^{c}$ LXXIX, R. Bellegou, prebre, vroy est.

[folio 12, folio non écrit]

[folio 12 verso]

Amprès comptent et se chargent lesdits procureurs des robes des parroessiens de ladite parroesse que sont decebdez oudit an que doibvent robes à ladite eglise, savoir la meilleure robe que une, ainxin que ensuit :

premier,

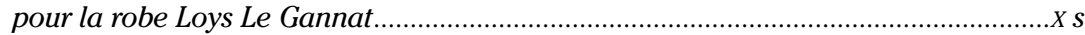

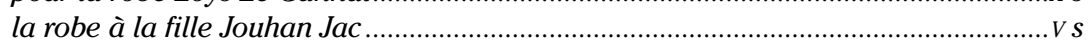

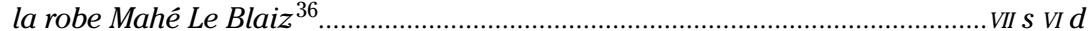

la robe de la veuffue Guillemot Crauzon.................................................................... $S$

la robe à la famme au Medec................................................................................... $s$ II $d$

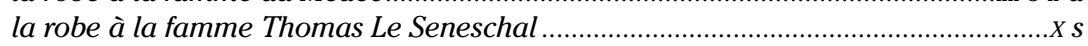

pour la robe à la famme Olivier Tarouale ....................................................... VII $s$ VI $d$

pour la robe Jehan Michel ............................................................................. $s$ VI $d$

pour la robe à la famme Guillaume Houat.................................................................. $s$

pour la robe Guillaume Le Couvier.....................................................................III $s$ IIII $d$

pour la robe à la femme Guillaume Sisaill................................................................. $s$

pour la robe à la femme Valentin Pierres......................................................... VIIII $s$ IIII $d$

pour la robe Thomas le Seneschal................................................................. $s$ VIII $d$

pour la robe à la famme Eon Goezval .......................................................... VII $s$ VI $d$

pour la robe du filz Jehan Mahé.................................................................................. $s$

pour la robbe Jehan Le Huedez................................................................................. $s$

Et ne se charge point de aucune anciennes robes des autres dont les noms sont desclerez cy davant es enterrements par ce qu'ils n'avoint que une robe et estoit pouvres [...] ont peut rien avoir.

[folio 13]

Emprès comptent et se chargent lesdits procureurs de la vente et livraison de la levee de deux eillez de marays en la saline Miroudrouc dont est paluyer Jehan Gego, eut trouvé deux mez et demy de sel venduz au pris de trante deux souls seiz deniers le mé, XVII moetz par mé, valloir audit pris ledit don rabatu, saixante saize soulz seiz deniers, dont alla pour le conduit II $s$ VI d par mé, qu'est VI $s$ III $d$. Reste, à cler, rabatu la part du paluier, et XX d de mise pour la reparacion.. .LI $s$ IIII $d$

36. Ce personnage est cité, $\mathrm{f}^{\circ} 10 \mathrm{v}^{\circ}$, sous le nom de Mahé le Lou (Blaiz = bleiz en breton, loup). La recension systématique des mots bretons contenus dans les archives d'époques médiévale et moderne de la sénéchaussée de Guérande est actuellement menée par G. Buron et donnera lieu à une ou plusieurs publications sur l'histoire de la langue bretonne entre Loire et Vilaine. 
Item se chargent de la levee d'un eillet de marays appartenant à ladite eglise en la saline Scaffne dont est paluier Jean Le Huedez qu'a valu, quicte de toute mise, la somme de XXII $s$ X

[en marge] à leur rapport et verificacion.

[folio 13 verso]

Item se chargent lesdits procureurs du louage de deux estaux appartenans à ladite eglise que ont esté louez pour $[\ldots]$

XI L XII $\mathbf{s}$ X d

Somme toute la charge desdits procureurs sept centz trente libvres doze soulz sept deniers, le XVIT jour de juign mil III C LXXIX, presens Jehan André, Guillaume Le Bosec, Jehan Jouan, Guillaume Jouan, Denis Quelo, Hervé Laic, Bernard Flahart, Olivier Rio, Jehan Maro et pluseurs autres.

N. Goic prebre commissaire signe

[folio 14]

Les mises, poimentz et descharges desdits procureurs premier,

Pour la procuration de la fabrique XVII $s$ VI $d$

[une première somme se montant à XXV s est barrée]

[en marge] alloué XVII $s$ VI d pour ce que mon seigneur de Nantes de present a baillé par edit et ordonnence que on ne baille que ladite somme de XVII $s$ VI $d$ pour seau et escripture de ladite procuracion, et le parssus refusé, sauff à leur faire raison dudit reffus lors qu'ilz aparestroint relacion de Jehan Pele qui leur bailla ladite procuracion.

Item à Eon Jouhano, clerc du vicaire, pour son salaire et despens de aler querir ladite procuracion à Nantes

[en marge] alloué à leur rapport et pour ce que mesmes les procureurs precedentz en ont compté de pareille somme pour ladite cause.

Item pour deux libvres d'encens que lesdits procureurs ont fait venir de Rennes par Jehan Gledel XIII $s$ IIII $d$

[en marge] alloué à leur rapport et que c'est chose neccessaire pour ladite eglise.

Item que ont poyé lesdits procureurs à frere Franczois Dignan, jacopin du couvent de Guerrande, pour et au nom dudit couvent la somme de LX s. Quelle somme avoit esté ordonnée et estre baillée au priour dudit couvent qui avoit presché en ladite parroesse tout au long du Quaresme et la Passion et la Resureccion oudit an, ainxi que apiert par lettre dudit commandement et quitance dudit Dignan et pour ce. ..$L X S$ [en marge] Alloué. Pour ceste foiz seulement, et par ce est deffendu es procureurs de ladite fabrique par le temps advenir de non en bailler plus pour le temps advenir sur paine de la perdre. Et dempuix à la visitation du Croesic de monseigneur de Nantes a esté deffence pour le temps avenir qu'il ne soit rien baillé esdit predicateur de l'argent de ladite fabrique.

Item à Jehan Onan et Eon Jouhanno pour la sonerie des cloches de ladite eglise pour ledit an comme meilleur marché en saesans..... 
[en marge] Alloué pour ce que a esté relaté par les assistans à ce compte ledit marché avoir esté fait pour ladite somme de $\mathrm{L}$ s avecques les declerez en l'article et que lesdits nommés confessé avoir esté poyés de ladite somme.

[folio 14 verso]

Item à Beiamin du Pont pour ses gages acoustumez de la sonerie des orgues pour ledit an ainsi que apiert par quitance de sa main. .$x L$

[en marge] Alloué. Ce qu'est le pris contenu en article lui est ordonné par gaiges par an et par ce que lesdits procureurs ont aparu quictence dudit du Pont, de Jouan, et dudit du Pont qu'elle est demeurée sur ce compte.

Item a dom Raoul Bellego pour ses gages davant raporté les enterrementz de ladite eglise et en passé les obligacions et pour avoir fait le raport du numbre des coupvrurs et des massons qui ont besoigné environ ladite eglise pour ledit an, la somme de XX $s$

[en marge] Alloué.

Item pour rente deue à la seignourie de Campsillon par cause de deux oiellez de marois en la saline Miroudrouc appartenant à ladite eglise pour ledit an. XII $d$

[en marge] Alloué comme es precedens.

Item pour le salaire de deux serviteurs qui ont gardé les tabliers des eglises Saint Grimolay et de Nostre Damme du Morier et en receu les deniers par ledit an ........XX $s$

[en marge] Alloué comme es precedentz.

Item à Olivier Le Pennec pour son salaire d'avoir servi environ ladite eglise, porté la banniere, cuilli et levé les robes des mortz, pour ledit an .......................................... $\mathrm{XV}$ [une somme de XVII $s$ VI $d$ est barrée]

[en marge] Alloué comme es precedentz.

Item suplient lesdits procureurs leur estre deschargé sur ce compte la somme de XX $s$ dont ils se sont chargez cy davant es rentes à deniers à prendre o les hers Eonet Herault sur la meson qui fut audit deffunct, pour ce que ladite rente n'est deue jucques après le deceix de la veuffve dudit Herault, combien qu'ilz s'en sont chargez affin qu'il en soit memoire comme es precedens compte, et pour ce. XXs

[en marge] Alloué pour le contenu de l'article.

\section{[folio 15]}

Item comme Jacquet Vinces et Guillo Cazdre precedans et derroins procureurs de ladite fabricque eussent fait feur et marché avecques Nicollas Le Texier et Guillaume Le Tili de faire et asseoirs une goutiere de pierre de taille entre les deux neffs de ladite eglise sellon ung certain devis qui en fut fait, pour en avoir la somme de neuf s sept d pour chacun pié de ladite goutiere et que dempuix ce ledit Nicolas Le Texer avoit fait et acompli ledit eupvre bien et deuement sellon le devis qui en avoit esté fait. Quelle goutiere contient de longueur cinquente neuff piéz et demy par le mesurage qui en a esté fait entre ledit Nicolas et lesdits procureurs, par et en la presence de Denis Quello, Hervé Layc, Jehan Conan, Jehan Le Boteuc, Jehan Mahé, Tanneguy Chalcot, Eon Le 
Texier et plusieurs autres ainsi que apiert par relacion desdits nomméz. Quelx cinquante neuff piéz et demy se montent audit pris la somme de vignt ouit livres diz soulz sept deniers dont avoit esté poié par lesdits procureurs precedants la somme de diz sept livres quatre soulz cinq deniers, ainsi restoit uncore audit Nicolas onze livres seix soulz deux deniers. Quelle somme lesdits procureurs luy ont fourny et poié ainsi que apert par quitance dudit maître Nicolas, et pour ce... $X I L$ VI $s$ II $d$

[en marge] Alloué. Et ont aparu le renable dudit feur et marché signé de Hervé Layc, Jouan, J. Le Boteuc, P. Rogon, Eon Le Boteuc que ledit eupvre estoit deuement fait et acompli et contenoit le numbre de piéz contenuz en l'article avecques quictence du poyement fait audit Le Texier pour il et ledit Le Tili. Quelx quictence, et relacion de renable sont demeurés sur le compte.

[folio 15 verso]

Item pour ce que par ledit feur et marché de asseoir ladite goutiere avoir esté dit que les procureurs de ladite fabrique devoint faire le massonnage soubz ladite goutiere. Lesdits procureurs en présence et du commandement de plusieurs des gens notables de la parrouesse firent marché avecques ledit Nicolas Le Texier de faire ledit massonnage par journées et fournir de pierres pour ce faire, pour en avoir ledit Nicolas pour chacune chartée de ladite pierre rendue sur le lieu la somme de quinze deniers et pour chacune journée audit Nicolas de sa personne III $s$ IX $d$ pour sallaire et despans et pour les autres massons troys soulz par journée et pour les serviteurs II $s$ IIII $d$ par journée ainsi que apiert par relacion signée de Jehan André et J. Conan. Et que dempuix ledit massonnage avoit esté fait par ledit Nicolas bien et deuement et en ont fait lesdits procureurs les poyments ainsi que la declaration ensuit :

[en marge] [...] est demeuré sur ce compte. Aussi ont aparu le raport de dom Raoul Bellego commis des fabriques et parroessiens à faire le rapport des charretées de pierre pour ledit eupvre avecques et des journées des maczons et serviteurs qui ont besoigné sur ledit eupvre. La relacion duquel raport est demeuré sur ce compte.

premier,

pour le vin du marché que en fut fait o ledit Nicollas en la meson Hervé de La Noé en présence de Jehan André, Guillaume Le Bosec, Denis Quello, Jehan Le Boteuc, Jehan Conan et plusieurs autres. . $s$ VIII $d$

[en marge] Alloué, au rapport et verification desdits procureurs.

[folio 16]

Item poierent audit Nicollas Le Texier pour quarante une chartée de pierre massonnage qui ont esté emploiées oudit eupvre, ainsi que apert par relacion de dom Raoul Bellego, commis quant affin de compter lesdites chartées, vallant audit pris de $\mathrm{XV} d$ chacune la somme de

[en marge] Alloué, pour ce que a esté aparu cy dessus, et la quictence pareillement en cest endroit aparue desdits maczons d'avoir esté poyez. Quelle quictence est demeurée sur ce compte.

Item pour trante sept journées de massons qui ont esté à faire ledit massonnage, en ce compte deux journées que ledit Nicolas a esté de sa personne, que se montent audit pris de III $s$ chacune journée et audit Nicolas III $s$ IX d par journée ainsi que apert par relacion dudit dom Raoul, la somme de..... CXII $s$ VI $d$ 
[en marge] Alloué, par ladite quictence dessus aparue, passé de Jouan et Marcraye.

Item pour les journées de XXXV serviteurs qui ont esté à servir lesdits massons audit pris de II $s$ IIII $d$ chacun... IIII $L$ I $S$ VIII $d$

[en marge] Alloué par la quictence cy dessus.

Item à Guillaume Jehan, febvre, pour le pointage des marteaux desdits massons en faisant ledit eupvre. XXIII $S$ IX $d$

[en marge] Alloué à leur rapport et verificacion et acquiteront vers ledit Jehan si faire ne l'ont.

[folio 16 verso]

Item poierent lesdits procureurs à Selvestre Guyomarch de Ruys pour saixante sept truellées de chaux qui ont esté emploiées oudit eupvre au pris de diz deniers chacune trullée que se montent audit pris comme apert par quitence dudit Guyomarc du quart jour de may l'an mil IIII ${ }^{c t}$ LXXVIII, la somme de.

[en marge] Alloué [...] esté employé oudit eupvre.

Item pour le sallaire de quatre mulles qui furent par une journée à porter ladite chaux du Croaesic à ladite eglise.

Item pour destramper ladite chaulx et pour le sallaire des femmes qui porterent l'eau et sable à mectre en ladite chaulx. IV $s$ II $d$

[en marge] Alloué à leur rapport et verificacion.

Item audit Selvestre Guyomarch par une autre foiz pour le feur quinze trullées de chaulx qui ont esté employées oudit eupvre sellon une quitance du XXVII de juign oudit an, la somme de.

[en marge] Alloué, par ce que ont aparu quictence dudit Guymar de ladite somme à la cause contenue en l'article et qu'ilz ont relaté et esté ledit numbre de chaux avoir esté pareillement employé oud eupvre.

\section{[folio 17]}

Item pour porter ladite chaulx du Croesic au bourg pour le sallaire de ouit mulles et deux hommes. Item pour destramper ladite chaulx, porter eau et sable Item poierent à ung nommé Mahé Perrot, marinier, pour son sallaire et despans d'avoir esté à Penerff achater et faire venir ladite chaulx par ce qu'il en y avoit quel neccessité à l'eure et convenoit tarder l'eupvre par deffault de chaulx, la somme de ...X $s$ Xd

[en marge] Alloué, à leur raport et verifficacion.

Item à Olivier Thefoche de Penerff pour trante deux trullées de chaulx pour ledit eupvre selon une quitance dudit Thefoche du quart jour de septembre oudit an, la somme de trante deux soulz.. XXXII $S$ 
[en marge] Alloué, par ce que ont aparu quictence dudit Thefoche quelle est demeurée sur ce compte et par ce que mesmes ont veriffié ladite chaux, avoir esté employée en ladite eglise.

Item pour la journée de trois mules qui furent à porter ladite chaux du Croesic à ladite eglise

[Cet article est d'une autre main]

[en marge] Alloué, à leur rapport et verificacion.

[folio 17 verso]

Item pour charoy d'une pipe de chaux du Croesic au bourg, le fust emprunter de monseigneur de Lauvrignac en atendant faire venir de l'autre chaux. XXII $d$

[en marge] Alloué, verifficacion.

Item à Georget Le Ray de Pennerff pour XXVIII truellées et demie de chaulx qui furent emploiees environ l'eupvre de ladite eglise la somme de XXVIII $s$ VI $d$

[en marge] Alloué [...] Comme apert par quitance dudit Georget [...] du XXe jour de septembre oudit an.

Item pour porter ladite chaux de Sterbriec à ladite eglise. IIII $S$ [Cet article est d'une autre main]

[en marge] Alloué.

Item fut fait marché par lesdits procureurs avecques Jehan Jarno, charpentier, de faire et asseoir les chevrons et coueaulx sur ladite eglise de chacun costé de la goutiere de pierre qui y estoit et de faire les boys pour les noes et toute autre charpentez environ ce nectessaires. Pour icelui Jarno en avoir desdits procureurs la somme de quatre soulz par journée pour sallaire et despans pour la somme dudit Jarno et pour les autres charpentiers de sa compaignie la somme de III s IIII d à chacun d'eulx par chacune journée pour sallaire et despans et quant affin de tailler et faire le rapport du numbre desdites journées avoit esté commis dom Raoul Bellego. Et y ont besougné lesdits Jarno et ses gens par XLV journées sellon que apert par relacion dudit dom Raoul.

[en marge] Alloué, pour les causes cy après declerées ou feillet prouchain.

[folio 18]

Queulx XLV journées, comme y comprins XV journées que ledit Jarno y avoit besougner de sa personne, se montent audit pris, la somme de VIII L. Quelle somme lesdits procureurs ont poiée audit Jarno ainsi que apert par quitance dudit Jarno et pour ceVIII L

[en marge] Par ce que a esté relaté par les assistans sur ce compte ledit marché avoir esté fait ovecques ledit Jarno [...] lesdits gens au pris contenu en l'article et qu'ilz ont aparu relacion dudit Jarno [...] somme de VIII L, avecques la relacion de dom Raoul Bellego, commis des procureurs [...] parroessiens pour compter les journées que ledit Jarno et ses gens eussent [...] dudit eupvre quelle relacion et quictence dudit poyment sont demouréz sur [...] 
Item poierent lesdits procureurs audit Jehan Jarno, charpentier, pour avoir fait deux lucarnes au desus de la goutière de ladite eglise par feur et marché en fait o ledit Jarno en presence de Jehan André, Denis Quello, Jacques Jouan, Jehan Conan, Jehan Le Boteuc et plusieurs autres, ainsi que apiert par relacion et quitance dudit Jarno, la somme de. .VII $L \times d$

[en marge] Alloué, et ont aparu le registre de feur et marché contenu en l'article avecques la quictence dudit Jarno d'avoir esté poyé de ladite somme queulx, passé par $J$. Conan et Jacquet Jouan, queulx sont demouréz sur ce compte.

[folio 18 verso]

Ensuyvent les mises faites par lesdits procureurs environ la couverture de ladite eglise et pour les matieres qui y ont esté employees,

premier

pour vignt sept milliers d'ardaise pierre d'Auvrigné et pour ung millier et demi de late pour ledit ediffice achatées de Pierre du Tay, marchant, demourant à Richebourg à Nantes, la somme de. LIII L XVII $s$ IIII $d$ ainsi que apert par relacion dudit du Tay.

Item pour la despence dudit Jehan Cabelguen qui fut à Nantes querir lesdites pierres et les achater, et mena en sa compagnie pour l'avertir à choisir lesdites pierres Guillaume Pihalle, couvreur, ouquel veage ilz furent par le temps de seix jours et des-

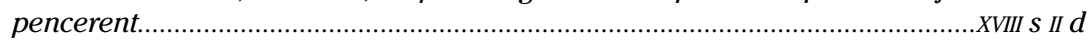
Item audit Pihalle pour son sallaire d'avoir esté o ledit Cabelguen à Nantes............. $s$

[en marge] Alloué, à leur rapport et verifficacion et qu'ilz ont aparu quictence dudit du Tay, quelles sont demourees sur ce compte.

[folio 19]

Item pour une barge que ledit Cabelguen loua pour le porter à Nantes.. $X I S$

[en marge] Alloué, à leur rapport et veriffication

Item poia pour deux sommes de clouz achactez de Jehan Ernaud, savoir clouz lateratz et de tache ou pris de $V \mathrm{~L}$ le millier ainsi qui aparert par relacion dudit Ernaud la somme de. IIII $L$ XII $S$

[en marge] Alloué, pareillemant et ont aparu quictence de Jehan Ernaud, quelle est demeurée sur ce compte.

Item pour deux mallectes à porter ledit clouz....... . II $S$

[en marge] Alloué, à leur rapport et verifficacion.

Item pour le fret d'une escaffe d'Abereledut qui porta partie de ladite pierre au Croesic, savoir, $V$ milliers et demy millier de late. ..XXXV $s$ Item pour le fret d'une autre escaffe d'Auray que porta partie de ladite pierre au Poulguen. XXXVS

[en marge] Alloué, comme devant. 
[folio 19 verso]

Item à Eon Le Lozdrec de Peherac pour le fret de son escaffe d'avoir aporté treize milliers et demy de pierre ardaise et ung millier de late de Nantes jucques au Croesic, la somme de.

comme aparert par quitance dudit Lozdrec.

Item pour descharger ladite pierre de l'escaffe dudit Le Lodrec et les arimer sur le cay du Croesic.

Item pour descharger ladite escaffe de pierre qui deschargea au Poulguen et pour les arimer sur ledit cay ..........................................................................................III $s$ II $d$ Item pour descharger la pierre de ladite escaffe d'Abereledut que deschargea au Croesic et pour les arimer sur le cay dudit lieu. II $s$ XI $d$

[en marge] Alloué [...] quelle est demeuré sur ce compte.

[folio 20]

Item à Symon Jouhanno et à Pezdron Jouhanno et à Jacob Jouhanno pour leur sallaire d'avoir porté les pierres d'ardaise, qui furent deschargées audit lieu du Poulguen, au bourg. $X V s$ II $d$

[en marge] Alloué, et ont aparu quictence dudit Jouhanno.

Item à Guillaume Alaire pour son sallaire d'avoir cherreay seix charetées pierre ardayse et une charetée late, du Croesic au bourg que feurent deschargées de ladite escaffe de Bereledut. XII $s$ VII $d$

[en marge] Alloué, et acquieront se fait ne l'ont.

Item ausdits Symon Jouhanno et Pedron Jouhanno pour le sallaire d'avoir cherreoy traize milliers et demy de pierre, du Croesic au bourc que furent deschargées de l'escaffe Eon Le Lozdrec, par marché fait o lesdits nomméz comme apert par quitance desdits nomméz, la somme de. $X X V S$

[en marge] Alloué comme devant.

III ${ }^{\mathrm{xx}}$ VI L XVIII S IX d

[folio 20 verso]

Item fut ledit Cabelguen audit lieu de Nantes pour faire venir, partie de ladite pierre d'ardaise pour ladite eglise, quelle il chargea en ladite escaffe au Lodrec et despancza tant en passages que en mises à charger ladite pierre la somme de XV $S$

[en marge] Alloué, rapport, veriffication

Item ont poié lesdits procureurs à Guillaume Gourel, couvreur pour avoir besougné environ la couverture de ladite eglise pour luy et autres couvreurs qui ont esté par le temps de II 't LIIII journées, ainsi qu'il apert par relacion de dom Raoul Bellego, commis desdits parrouessiens quant affin de faire le rapport desdits journées desdits oupvriers gaignans par chacun jour pour sallaire et despans la somme de II $s$ XI $d$, que se montent audit numbre de journées, la somme de XXXVII L $X d$ Comme apert par quittance dudit Gourel. 
[en marge] [...] ledit marché a esté fait ovecques ledit Gourel long temps par avant avoir encommancé ladite coupverture au pris ainsi contenu en l'article et par ce que a esté aparu par relacion de dom Raoul Bellego commis à faire le rapport des journées que feroit ledit Gourel sur ledit eupvre, ledit Gourel avoit fait ledit numbre de journées rapportées oudit article, quelles journées se montent au pris contenu en l'article ladite somme et par ce mesme lesdits procureurs ont eu quictence comme ledit Gourel avoit esté poié de ladite somme. Quelle relacion et quictence sont demeurez sur ce compte, ladite quctence signée de Conan et $P$. Rogon.

\section{[folio 21]}

Item poierent lesdits procureurs pour quarante deux planches de fou, boys de Rouan, qui furent emploiées à lembruncher les lucarnes de ladite eglise environ les noes valant à VII d chacune planche.............................................................................XIIII $s$ VI $d$ Item pour le sallaire d'un charrecte qui porta lesdites planches au bourc................XII d Item pour dix sept fustz de pipe qui furent emploiéz environ les noes de ladite eglise et à lembruncher lesdites lucarnes la somme de ...............................................XXXIV $s$ Item pour le sallaire d'une charrecte qui porta lesdits fustz au bourc ........................XIX d Item poierent lesdits procureurs pour le cherroy de quatre mastz de navires et cinq grandes bourlingues qui furent empruntées du Croaesic pour faire les chaffaults du massonage et de la charpenterie de ladite eglise et les rendre du bourc au Croaesic. XII $s$ VI $d$

[en marge] Alloué a leur rapport et verifficacion.

[folio 21 verso]

Item pour deux cordes de bouille qui furent achattées pour lier les chaffaults de ladite

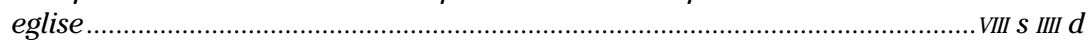
Item pour une autre corde qui fut faite pour le guindage des pierres et boays de l'édiffice dudit eglise, quelle corde a esté mise es cloches d'icelle eglise ....................III $s$ IX $d$ Item poierent pour le deché d'une grande haussiere qui fut empruntée de Nicolas Le Leisour pour faire le guindage des grosses pierres de la goutiere de ladite eglise, la somme de.

Item à Jehan Gledel pour XII miliers de cheville et pour vict et demy de clou de tillat $V I^{c t}$ et demy de clou despuer qui ont esté emploiés environ la couverture de ladite eglise ainsi que apiert par rellacion dudit Gledel. ..XXXII $s$ [Cet article est d'une autre main]

[en marge] Alloué, et a aparu quictence dudit Gledel et est demeuré sur ce compte.

[folio 22]

Item pour descendre les veilles pierres d'ardayse de la despouille de ladite eglise et trier à part les bonnes pierres et les arumer ou cymetiere et porté le parsus hors avecques le demourant des actreitz des massons, la somme de .............................II $s$ Item pour curer et necteer l'église et le cymetiere à la venue de monseigneur de Nantes

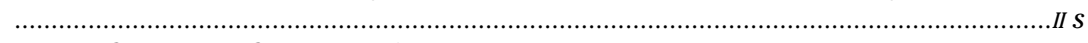
Item à Guillaume Gourel qui fut par une journée ovecques les charpentiers au commancement de la charpenterie de ladite eglise pour les advertir.. II $s$ VI $d$

[en marge] Alloué, a leur rapport et veriffication.

[folio 22 verso]

Item pour massonner une berche du cymetiere de ladite eglise Saint Grimolay devers la meson dom Jehan Le Maller.. 
Item à Guillaume Le Paintre pour son sallaire d'avoir reparé et mis aparoit ung pertuys qui estoit en la vitre de la chappelle de Nostre Damme en ladite eglisse Saint Grimolay et pour avoir reparé une fenestre de ladite eglise au dessus de la grant vitre par dessus la voulte ................ XI $s$ VIII Item pour doze saintures à saindre les chappelains à la messe.................................. $\mathrm{s}$

[en marge] Alloué pour ce que a esté relaté par dom Guillaume Le Bourdiec, segretain de la dite eglise, lesdites saintures estre en ladite eglise et pour ce que lesdits procureurs ont verifié icelle avoir cousté ladite somme.

[folio 23]

Item poierent à Jehan Cadoret pour deux petiz orceulz d'argent pour ladite eglise en présence et du commandement de monseigneur de Lauvrignac, Jehan André et plussieurs autres ainsi que apert par relacion [...]

[en marge] Alloué et est aparu quictence dudit Cadoret d'estre poyé de ladite somme et demeurée sur ce compte.

Item que ont poyé à Allain Gaultier [...] avoir lembrunché la neff de l'eglise Sainct Grimolay [...] chapelle Nostre Damme en ladite eglise [...] où avoit besoign de reparacion environ [...] de ladite eglise par fuer et marché en fait et banni. Quel fuer lui estoit demouré comme derrain desboutant comme apiert par rellacion et qucttance, la somme de $L V[\ldots]$

[en marge] Alloué, et ont aparu le devis du feuer fait touchant ce avecques ledit Gaultier et quictence d'icelui, dict avoir esté poyé et demeurée sur ce compte.

Item à Jehan Gledel pour le clou qui fut employé environ ledit eupvre comme apiert par relacion dudit Gledel, la somme de VI $s$ VIII $d$

[en marge] Alloué, o leur rapport et verifficacion et acquitteront si fait ne l'ont.

[folio 23 verso]

Item ont poyé lesdits procureurs à Simon Uzel pour avoir fait et assis la verrine de la lucarne de ladite eglise sur la goutiere de ladite eglise, la prouchaine lucarne de la goutiere parachevée faite ledit Simon pour le pris de cinq soulz chacun pié [...] quelle verrine contient dix ouit piéz [...] comme apiert par relacion IV LXS

[en marge] Alloué, ont aparu le feur et ovecques ce que ledit Uzel la quictence dudit estre poyé $[\ldots]$

[...] Le Guiader pour avoir fait es [...] fermeture de la verrine de ladite [...] avecques dont Denis Quello [...], la somme de

[en marge] $[\ldots]$

Item à Guillaume Le Paintre pour la verine de la louere de ladite eglise qui est l'endroit du letrin.

[en marge] Alloué [...]

Item à Guillaume Gourel pour faire ladite louere III $s$ IX $d$ 
[en marge] Alloué.

Item pour une corde pour la petite cloche de ladite eglise et pour ung rebant pour ladite louere...

[en marge] Alloué, o leur rapport et verifficacion et qu'il a esté relaté par les assistans ladite corde avoir esté atachée à ladite cloche.

[folio 24]

Item ont poié lesdits procureurs [...] pour le louage de la meson de [...] pierres d'ardayse de ladite [...] long temps pour compte [...] Jouhanno dont y a quitance [...]

[en marge] Alloué par ce que a esté relaté par les assistants ledit appointement avoir esté $[. .$.$] ovecques ledit Jouhanno à ladite somme par la cause contenue en l'article$ et ont aparu quictence dudit Jouhanno d'en avoir esté [...] demourée en ce compte.

Item ont poié lesdits procureurs pour [...] de ostade noire que [...] à faire ung chasuble damaires [...] eglise par le commandement de plusieurs [...] de ladite paroesse et neuf estolles et $[\ldots] x$ fanons ................................................................................ $L$ Item pour les orfrais desdits ornemans, savoir, pour lesdits chasuble, damaires, et chappe faiz faire à Rennes par Jehan Gledel ainxin que apiert par relacion dudit Gledel,

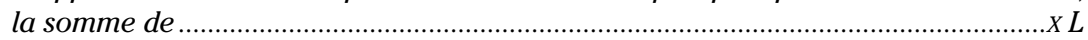
Item audit Gledel pour XV aulnes de bougrain de Paris que ont esté emploiez à doubler lesdits ornementz et lesdites estoles et fanons au pris de III $s$ IX $d$ chacune aulne...........LX $s$ Item pour neuff onces de fil noir que ont esté prins dudit Gleder pour coudre les ornementz au pris de VII d la once. V $S$ III $d$

[en marge] Alloué et a esté relaté par les assistans sur ce compte lesdits articles contenir verité.

III $L X V I[\ldots]$

[folio 24 verso]

[...] laz de fil qu'ils ont esté emploiéz........................................................................ $s$

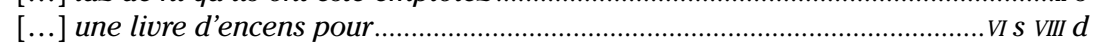

[...] emploié à faire lesdits ornements [...] Guillo Bretin par Lorans Hamon ...IX s IV d

$[\ldots]$ emploie à faire ......................................................................................... $s$ II $d$

$[\ldots]$ pour la faczon des ornementz.............................................................II $s$ VI $d$

[folio 25]

Item à Jehan Le Botec po[...] l'apointement que a esté fait [...] de Baz touchant le priouré $[\ldots]$ eglise et le priouré que $[\ldots]$ dudit priour $[. .$.

[en marge] Alloué, et ont rendu ledit contract à Pezdron Le Caloch à present procureur pour en respondre.

Item pour les seaulx desdits $[\ldots]$

[en marge] Alloué, comme devant et que le contract est de heritaige.

Item que ont poié lesdits [...] pour les messes par lui faite [...] Landevennec ratiffier ledit $[\ldots]$ pour quant à le tenir ainsi que apert par [...] relacion dudit dom Jacob [...] 
[en marge] Alloué, et ont aparu garant signé et G. Le Bosec, Jehan Bouchart et plusieurs aultres avecques relacion dudit dom Jacob d'avoir eu ladite somme.

Item pour meptre et oter la tapicerie de ladite eglise durant ledit an [...]

[en marge] Alloué, pour ceste foiz.

Item le salaire de garder la table Nostre Damme du Morier pour ledit an.........XII $s$ VI $d$ Item pour le salaire de garder la table de religion de l'eglise monsieur Saint Grimolay

[Les deux précédents articles sont barrés] VII $s$ VI $d$

[en marge] Alibi cy davant.

[folio 25 verso]

Item ont poyé lesdits procureurs [...] feste du Sacrement [...]. XIII $S$ IV $d$

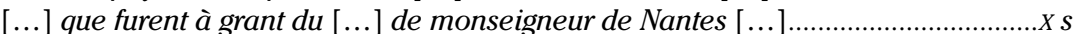

$[\ldots]$ ceulx qui porterent la $[\ldots]$ à la Trenité $[\ldots]$.................................................... $s$

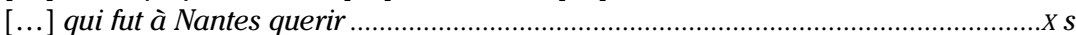

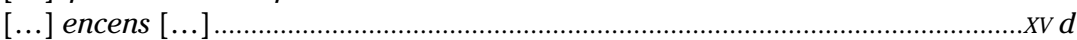

[...] l'arrolement de ce compte.................................................................................. $s$ Item supplient leur estre alloué les biens et thresors de la dite eglise comme ornementz, galices, ymaiges d'or et d'argent, livres et aultres biens dont ils se sont chargés au premier article de ce compte pour ce que ont rendu lesdits biens à present l'un des procureurs de ladite fabrique.

[en marge] Alloué, ledit Pierres Le Caloch ${ }^{37}$ a esté present et a comparu, avoir eu lesdits biens ainsi qu'ilz sont contenuz en l'inventaire cy devant et à present en respondre.

[folio 26]

Item pour la des[...] deux jours par ce [...] fait avecques Jehan [...] ladite eglise et le [...] de la somme de $[. .$.

La mise et descharge de [...] la somme de [...]

Et leur charge $[\ldots]$

Deducion faicte de mise [...] et Benabès Jouhanno la somme [...] livres monnoie. Quelle somme leur est [...] Calouch et Jacob Le Laisour à present procureurs [...] prouchaine venente pour tout [...] par leurs serments ainsi faire et $[\ldots]$ maire forme de contracts, errour de compte [...] en touz endroictz. Ce compte fait et conclut par Nicholas [...] vicaire et commissaire quant adce en la presence de Jean Jouhan, Jehan André, Mahé Legliff, Guillaume Jouhan, Hervé Layc, Jehan Conan, Jehan Le Boteuc, Symon Le Rouxeau [...] vigntyesme jour de juign mil IIIIt saixante [...]

[signatures]

M. Le Gliff, J. Le Boteuc, Nic. Goïc, commissaire, signe, J. Conan, G. Jouhan, Hervé Layc.

[folio 26 verso]

Bonabès $[\ldots]$ sadite $[\ldots]$ le comm $[\ldots]$ ils ont esté $[\ldots]$

$[\ldots]$ autrement la famme $[\ldots]$ la somme de XX $s[\ldots]$

37. Dans les autres feuillets du compte, le prénom du personnage apparaît sous sa forme bretonne, Pezdron, Pedron, $\mathrm{f}^{\circ} 25 \mathrm{v}^{\circ}$. 
$[\ldots]$ que lesdits procureurs de la somme de [...] de la veuve Eon Foenant [...] esté ordonné par les asistans [...] compte que lesdits procureurs en eussent esté deschargez [...] en leur mise par certaine donayson que ledit Eon Foeanant a donné à ladite eglise pour poiement dudit enterrement et n'en ont point lesdits procureurs demandé descharge en mise et pour ce suplient ladite somme leur estre deschargee et pour ce

[folio 27]

$[\ldots]$

\section{RÉSUMÉ}

Le document révèle la gestion d'une fabrique, personne morale gérée par des laïcs, représentée par des procureurs, encadrée par l'autorité épiscopale. Les procureurs assurent l'entretien des bâtiments, du mobilier cultuel, engagent les travaux décidés et plus généralement gèrent le temporel de l'église. En cela, ils concourent à l'intérêt général. Cependant, les décisions ne relèvent que d'un groupe d'hommes formant les nobles, bourgeois et gens notables de la paroisse de Batz, assimilable au général de paroisse dont l'administration épiscopale paraît soucieuse d'assurer l'autonomie par rapport au pouvoir civil, celui lié à la municipalité croisicaise. Le compte livre encore une série d'informations sur la vie religieuse, sociale et économique de la paroisse.

\section{ABSTRACT}

The document puts into evidence the way a fabrique is managed; this legal entity, ruled by laymen, is represented by procurators and placed under the control of the episcopal authority. The procurators are in charge of the maintenance of buildings as well as religious furniture and implements; they undertake the programmed work projects. As a whole, they manage the temporal features of the church, thus contributing to the general interest. Yet, all decisions are taken by a small party of men belonging to the nobles, bourgeois et gens notables de la paroisse de Batz, roughly identical to the general of the parish, which the episcopal administration looks very anxious to keep independent from the civil power linked with Le Croisic town council. The account book contains still more information about the religious, social and economical life of the parish. 
